\title{
Non-conformal supercurrents in six dimensions
}

\author{
Sergei M. Kuzenko, ${ }^{a}$ Joseph Novak $^{b}$ and Stefan Theisen ${ }^{b}$ \\ ${ }^{a}$ School of Physics and Astrophysics M013, The University of Western Australia, \\ 35 Stirling Highway, Crawley W.A. 6009, Australia \\ ${ }^{b}$ Max-Planck-Institut für Gravitationsphysik, Albert-Einstein-Institut, \\ Am Mühlenberg 1, D-14476 Golm, Germany \\ E-mail: sergei.kuzenko@uwa.edu.au, joseph.novak@aei.mpg.de, \\ stefan.theisen@aei.mpg.de
}

ABSTRACT: Non-conformal supercurrents in six dimensions are described, which contain the trace of the energy-momentum tensor and the gamma-trace of the supersymmetry current amongst their component fields. Within the superconformal approach to $\mathcal{N}=(1,0)$ supergravity, we present various distinct non-conformal supercurrents, one of which is associated with an $\mathcal{O}(2)$ (or linear) multiplet compensator, while another with a tensor multiplet compensator. We also derive an infinite class of non-conformal supercurrents involving $\mathcal{O}(n)$ multiplets with $n>2$. As an illustrative example we construct the relaxed hypermultiplet in supergravity. Finally, we put forward a non-conformal supercurrent in the $\mathcal{N}=(2,0)$ supersymmetric case.

KEywords: Extended Supersymmetry, Supergravity Models, Superspaces

ARXIV EPRINT: 1709.09892 


\section{Contents}

1 Introduction $\quad 1$

2 Non-conformal $\mathcal{N}=(1,0)$ supercurrents $\quad 4$

2.1 Non-conformal supercurrents in Minkowski superspace 5

2.2 The non-conformal supercurrent based on a compensating $\mathcal{O}(2)$ multiplet $\quad 9$ 2.2.1 An example: Abelian gauge theory coupled to an $\mathcal{O}(2)$ multiplet 10

2.3 The non-conformal supercurrent involving an $\mathcal{O}(4)$ multiplet $\quad 11$ 2.3.1 An example: the relaxed hypermultiplet 12

$\begin{array}{lll}2.4 & \text { Further generalisations } & 14\end{array}$

3 The supercurrent associated with the dilaton-Weyl multiplet $\quad 15$

3.1 An example: non-abelian gauge theory involving a compensating tensor $\begin{array}{ll}\text { multiplet } & 16\end{array}$

$\begin{array}{ll}3.2 & \text { The dilaton-Weyl multiplet } \\ & 18\end{array}$

4 The $\mathcal{N}=(2,0)$ non-conformal supercurrent 20

5 Discussion $\quad 22$

A The geometry of $\mathcal{N}=(1,0)$ conformal superspace in six dimensions 24

$\begin{array}{ll}\text { B The prepotential for the } \mathcal{O}(2) \text { multiplet } & \mathbf{2 7}\end{array}$

$\begin{array}{ll}\text { C The Yang-Mills multiplet in conformal superspace } & 29\end{array}$

D A superform description for the $\mathcal{N}=(2,0)$ tensor multiplet and its $\begin{array}{ll}\text { deformation } & \mathbf{3 0}\end{array}$

\section{Introduction}

In supersymmetric field theory, the energy-momentum tensor belongs to a supermultiplet, called the supercurrent [1]. In the case of superconformal theories, the supercurrent multiplet is irreducible. It contains the energy-momentum tensor $T_{m n}$, the spinor $Q$-supersymmetry current $S_{m}$ and the $R$-symmetry current $j_{m}$, in conjunction with some additional components that are required in order to have an equal number of bosonic and fermionic components. ${ }^{1}$

\footnotetext{
${ }^{1}$ Those conserved currents, which correspond to the other continuous transformations in the superconformal group, are constructed from the conformal supercurrent and conformal Killing supervector fields [2].
} 
For theories without superconformal symmetry, the supercurrent multiplet is reducible, as a rule. ${ }^{2}$ The point is that the energy-momentum tensor is no longer traceless, and its trace $T^{m}{ }_{m}$ may belong to a smaller supermultiplet embedded in the non-conformal supercurrent. This trace supermultiplet also contains the $\gamma$-trace of the $Q$-supersymmetry current, $\gamma^{m} S_{m}$, as well as the divergence of the $R$-symmetry current, $\partial^{m} j_{m}$, if the $R$ symmetry current is not conserved.

An example worth recalling is the supercurrent multiplet [1] corresponding to $\mathcal{N}=1$ Poincaré supersymmetry in four dimensions (4D). The conformal supercurrent is described by a real axial vector superfield, $J_{m}=\bar{J}_{m}$, constrained by

$$
\bar{D}^{\dot{\alpha}} J_{\alpha \dot{\alpha}}=0 \text {. }
$$

The non-conformal supercurrent proposed by Ferrara and Zumino [1] is

$$
\bar{D}^{\dot{\alpha}} J_{\alpha \dot{\alpha}}=D_{\alpha} X, \quad \bar{D}_{\dot{\alpha}} X=0 .
$$

Here $X$ is the trace supermultiplet. ${ }^{3}$

Unlike the conformal supercurrent, its non-conformal counterpart is not unique. The reason for this is that there may exist several inequivalent trace supermultiplets supported by different supersymmetric field theories [7]. For instance, another example of $4 \mathrm{D} \mathcal{N}=1$ non-conformal supercurrents is [7-9]

$$
\bar{D}^{\dot{\alpha}} J_{\alpha \dot{\alpha}}=\chi_{\alpha}, \quad \bar{D}_{\dot{\beta}} \chi_{\alpha}=0, \quad D^{\alpha} \chi_{\alpha}=\bar{D}_{\dot{\alpha}} \bar{\chi}^{\dot{\alpha}}
$$

Here the trace supermultiplet $\chi_{\alpha}$ is a vector multiplet.

Similar to the energy-momentum tensor, which is the source of gravity, the supercurrent is the source of supergravity [10-12]. For a given Poincaré supergravity theory, there often exist several off-shell formulations leading to the same dynamical system on shell. However, different off-shell formulations for supergravity lead to different non-conformal supercurrents. In the case of $4 \mathrm{D} \mathcal{N}=1$ supergravity, for instance, the supercurrent multiplet (1.2) is associated with the old minimal formulation [13-15], while the conservation equation (1.3) corresponds to the new minimal formulation [8].

The connection between the non-conformal supercurrents and different off-shell formulations for supergravity becomes more apparent in the Weyl-invariant (or conformal) approach to supergravity. Before discussing the supersymmetric case, it is instructive to recall the Weyl-invariant formulation for gravity. Consider a system of matter fields $\varphi^{i}$ coupled to the gravitational field. In the approach of [16-18], the gravitational field is described by the metric $g_{m n}$ and the conformal compensator $\phi$, the latter being a nowhere vanishing scalar field. ${ }^{4}$ The action must be invariant under general coordinate and Weyl

\footnotetext{
${ }^{2}$ There exist counter-examples in five and six dimenisons [3, 4].

${ }^{3}$ Since $D^{2} X-\bar{D}^{2} \bar{X}=-2 \mathrm{i} \partial_{\alpha \dot{\alpha}} J^{\dot{\alpha} \alpha}$, the chiral scalar $X$ in (1.2) is an example of the so-called three-form multiplet [5] (see [6] for a review). For instance, in quantum supersymmetric Yang-Mills theories it holds that $\langle X\rangle=\kappa \operatorname{tr}\left(W^{\alpha} W_{\alpha}\right)$, where $k$ is a real parameter, and $W_{\alpha}$ the chiral field strength of the Yang-Mills supermultiplet.

${ }^{4}$ As in [16-18], our discussion here is restricted to the 4D case, but generalisation to higher dimensions is obvious.
} 
transformations,

$$
\delta g_{m n}=\nabla_{m} \lambda_{n}+\nabla_{n} \lambda_{m}-2 \sigma g_{m n}, \quad \delta \phi=\lambda^{m} \nabla_{m} \phi+\sigma \phi,
$$

augmented by certain transformations of the matter fields. Consider the matter action

$$
S_{\mathrm{M}}=\int \mathrm{d}^{4} x \sqrt{-g} \mathcal{L}\left(\varphi^{i} ; g_{m n}, \phi\right) .
$$

If the metric and the compensator acquire arbitrary infinitesimal displacements, $g_{m n} \rightarrow$ $g_{m n}+\delta g_{m n}$ and $\phi \rightarrow \phi+\delta \phi$, the action varies as

$$
\delta S_{\mathrm{M}}=\int \mathrm{d}^{4} x \sqrt{-g}\left\{\frac{1}{2} T^{m n} \delta g_{m n}+T \delta \ln \phi\right\},
$$

where $T^{m n}$ denotes the energy-momentum tensor of the system. If the matter fields are chosen to obey their equations of motion, $\delta S_{\mathrm{M}} / \delta \varphi^{i}=0$, the conditions of invariance of $S_{\mathrm{M}}$ under the local transformations (1.4) are

$$
\begin{aligned}
\nabla_{n} T^{m n} & =T \nabla^{m} \ln \phi, \\
g_{m n} T^{m n} & =T .
\end{aligned}
$$

The Weyl invariance may be used to impose a condition $\phi=$ const, in which case eq. (1.7a) turns into

$$
\nabla_{n} T^{m n}=0
$$

which is the standard conservation equation.

In analogy with the Weyl-invariant formulation for gravity [16-18], Poincaré or antide Sitter supergravity theories may be formulated as conformal supergravity coupled to a compensating supermultiplet [19, 20]. Unlike gravity, however, supergravity generally allows for several choices of conformal compensator that differ in their auxiliary fields. It turns out that different conformal compensators lead to different off-shell supergravity theories and, as a consequence, to different supercurrent multiplets. For instance, the conservation equation (1.2) of the old minimal formulation of supergravity corresponds to a compensating chiral scalar multiplet, while the conservation equation (1.3) of the new minimal formulation of supergravity corresponds to a compensating tensor multiplet.

For $6 \mathrm{D} \mathcal{N}=(1,0)$ supersymmetry, the conformal supercurrent was described more than thirty years ago [4]. However, to the best of our knowledge, no classification of non-conformal supercurrents has been given. The only known non-conformal $\mathcal{N}=(1,0)$ supercurrent was proposed by Manvelyan and Rühl [21]. It proves to be a $6 \mathrm{D}$ analogue of the $4 \mathrm{D} \mathcal{N}=2$ non-conformal supercurrent introduced by Stelle [22]. The latter obeys the conservation equation

$$
\bar{D}^{i j} J=\frac{1}{5} \bar{D}_{k l} \mathbb{L}^{k l i j}, \quad \bar{D}^{i j}:=\bar{D}_{\dot{\alpha}}^{i} \bar{D}^{\dot{\alpha} j}
$$

where $J=\bar{J}$ denotes the $\mathcal{N}=2$ supercurrent $[23,24]$. The trace supermultiplet $\mathbb{L}^{i j k l}=$ $\mathbb{L}^{(i j k l)}$ is real, $\overline{\mathbb{L}^{i j k l}}=\mathbb{L}_{i j k l}$, and is subject to the analyticity constraints defining an $\mathcal{O}(4)$ multiplet,

$$
D_{\alpha}^{(i} \mathbb{L}^{j k l m)}=\bar{D}_{\dot{\alpha}}^{(i} \mathbb{L}^{j k l m)}=0
$$


It was shown in $[25,26]$ that the conservation equation (1.9) naturally occurs in theories which couple to the $\omega$-hypermultiplet compensator $[27,28]$ within the harmonic superspace approach to $4 \mathrm{D} \mathcal{N}=2$ supergravity (see [29] for a review).

The purpose of this paper is twofold: (i) to derive various consistent $6 \mathrm{D}$ non-conformal supercurrents; and (ii) to lift them to an arbitrary curved conformal supergravity background with a conformal compensator. As a consequence, all non-conformal supercurrents may be classified by the choice of compensating conformal supermultiplet.

This paper is organised as follows. In section 2 we present an infinite family of $6 \mathrm{D} \mathcal{N}=$ $(1,0)$ non-conformal supercurrents involving $\mathcal{O}(n)$ multipletsfor $n>1$ and we illustrate a couple of them by analysing the equations of motion for certain models. ${ }^{5}$ Section 3 is devoted to the special case of using an $\mathcal{N}=(1,0)$ tensor multiplet as a compensator. We put forward a non-conformal supercurrent for the $\mathcal{N}=(2,0)$ case in section 4 . Finally, we discuss our results in section 5 .

We have included a number of technical appendices. Throughout this paper we will make use of the superspace formulation of conformal supergravity known as $6 \mathrm{D} \mathcal{N}=(1,0)$ conformal superspace [41]. Therefore, we provide the salient details of conformal superspace in appendix A. Appendix B is devoted to the prepotential description of the $\mathcal{O}(2)$ (or linear) multiplet. In appendix $\mathrm{C}$, we summarise the description of the Yang-Mills multiplet in conformal superspace. Finally, we give a superform description of the $\mathcal{N}=(2,0)$ tensor multiplet and its deformation in appendix D.

\section{Non-conformal $\mathcal{N}=(1,0)$ supercurrents}

In $6 \mathrm{D} \mathcal{N}=(1,0)$ supergravity, the conformal supercurrent $J$ is a primary superfield of dimension +4 ,

$$
\mathbb{D} J=4 J, \quad S_{i}^{\alpha} J=0,
$$

which satisfies the conservation equation [41]

$$
\nabla^{\alpha i j k} J=0, \quad \nabla^{\alpha i j k}:=\frac{1}{3 !} \varepsilon^{\alpha \beta \gamma \delta} \nabla_{\beta}^{(i} \nabla_{\gamma}^{j} \nabla_{\delta}^{k)} .
$$

In the flat superspace limit, this equation reduces to the one originally given in [24].

In the presence of a conformal compensator the conservation equation (2.2) is deformed to

$$
\nabla^{\alpha i j k} J=A^{\alpha i j k},
$$

where $A^{\alpha i j k}$ is a primary superfield of dimension $\frac{11}{2}$. Using the identity

$$
\nabla_{\alpha}^{(i} \nabla^{\beta j k l)}=\frac{1}{4} \delta_{\alpha}^{\beta} \nabla_{\gamma}^{(i} \nabla^{\gamma j k l)}
$$

\footnotetext{
${ }^{5}$ The $\mathcal{O}(n)$ multiplets are well known in the literature on supersymmetric field theories with eight supercharges in diverse dimensions. For $4 \mathrm{D} \mathcal{N}=2$ Poincaré supersymmetry, general $\mathcal{O}(n)$ multiplets, with $n>2$, were introduced in [30-33]. The case $n=4$ was first studied in [34]. The terminology "OOO(n) multiplet" was coined in [35]. As $6 \mathrm{D} \mathcal{N}=(1,0)$ superconformal multiplets, their complete description was given in [36] following the earlier approaches in four and five dimensions [37-40].
} 
it can be checked that $A^{\alpha i j k}$ must satisfy the following integrability condition:

$$
\nabla_{\alpha}^{(i} A^{\beta j k l)}=\frac{1}{4} \delta_{\alpha}^{\beta} \nabla_{\gamma}^{(i} A^{\gamma j k l)}
$$

In order to guarantee the existence of a conserved supersymmetry current and energymomentum tensor, the integrability condition (2.5) has to be accompanied with some additional requirements on the structure of $A^{\alpha i j k}$. To understand this in more detail, it is worth analysing the deformed conservation equation (2.3) in Minkowski superspace and uncover the corresponding component structure. In what follows, we will refer to the superfield $A^{\alpha i j k}$ as the trace superfield since in general it gives a trace contribution to the energy-momentum tensor, while $J$ only contains a symmetric traceless contribution.

\subsection{Non-conformal supercurrents in Minkowski superspace}

In this subsection we will make use of the spinor derivatives for $6 \mathrm{D} \mathcal{N}=(1,0)$ Minkowski superspace, $D_{\alpha}^{i}$, which satisfy the anti-commutation relation

$$
\left\{D_{\alpha}^{i}, D_{\beta}^{j}\right\}=-2 \mathrm{i} \varepsilon^{i j} \partial_{\alpha \beta}
$$

and commute with partial vector derivatives, $\left[\partial_{a}, D_{\alpha}^{i}\right]=0$.

We now analyse the component structure of the superfields $J$ and $A^{\alpha i j k}$ subject to the general constraints (2.3) and (2.5) in the flat-superspace case. ${ }^{6}$ Taking successive spinor derivatives of the trace superfield $A^{\alpha i j k}$, one finds ${ }^{7}$

$$
\begin{aligned}
D_{\alpha}^{i} A^{\beta j k l}= & \delta_{\alpha}^{\beta} A^{i j k l}+\varepsilon^{i(j} A_{\alpha}{ }^{\beta k l)}+\delta_{\alpha}^{\beta} \varepsilon^{i(j} A^{k l)}, \quad A_{\alpha}{ }^{\alpha i j}=0 \\
D_{\alpha}^{p} A^{i j k l}= & \varepsilon^{p(i} \Lambda_{\alpha}{ }^{j k l)} \\
D_{\alpha}^{k} A^{i j}= & \frac{\mathrm{i}}{2} \partial_{\alpha \beta} A^{\beta i j k}+\varepsilon^{k(i} \Lambda_{\alpha}^{j)} \\
D_{\alpha}^{k} A_{\beta}{ }^{\gamma i j}= & -\delta_{\alpha}^{\gamma} \Lambda_{\beta}^{i j k}+\frac{1}{4} \delta_{\beta}^{\gamma} \Lambda_{\alpha}{ }^{i j k}-\frac{4}{3} \varepsilon^{k(i} \delta_{\alpha}^{\gamma} \Lambda_{\beta}^{j)}+\frac{1}{3} \varepsilon^{k(i} \delta_{\beta}^{\gamma} \Lambda_{\alpha}^{j)}+\varepsilon^{k(i} \Lambda_{\alpha \beta}{ }^{\gamma j)} \\
& +2 \mathrm{i} \partial_{\alpha \beta} A^{\gamma i j k}-\frac{\mathrm{i}}{2} \delta_{\beta}^{\gamma} \partial_{\alpha \delta} A^{\delta i j k}, \quad \Lambda_{\alpha \beta}{ }^{\beta i j}=0, \quad \Lambda_{\alpha \beta}{ }^{\gamma i j}=\Lambda_{[\alpha \beta]}{ }^{\gamma i j} \\
D_{\alpha}^{l} \Lambda_{\beta}{ }^{i j k}= & 2 \mathrm{i} \partial_{\alpha \beta} A^{i j k l}+\varepsilon^{l(i} \mathcal{A}_{\alpha \beta}{ }^{j k)}, \quad \mathcal{A}_{\alpha \beta}{ }^{i j}=\mathcal{A}_{[\alpha \beta]}{ }^{i j}, \\
D_{\alpha}^{i} \Lambda_{\beta}^{j}= & \varepsilon^{i j} \mathcal{A}_{\alpha \beta}+\frac{\mathrm{i}}{2} \partial_{\gamma \alpha} A_{\beta}^{\gamma i j}-\frac{\mathrm{i}}{6} \partial_{\gamma \beta} A_{\alpha}^{\gamma i j}+\frac{4 \mathrm{i}}{3} \partial_{\alpha \beta} A^{i j}, \quad \mathcal{A}_{\alpha \beta}=\mathcal{A}_{[\alpha \beta]}, \\
D_{\alpha}^{i} \Lambda_{\beta \gamma}{ }^{\delta j}= & \frac{2}{3} \delta_{[\beta}^{\delta} \mathcal{A}_{\gamma] \alpha}{ }^{i j}+\delta_{\alpha}^{\delta} \mathcal{A}_{\beta \gamma}{ }^{i j}+4 \mathrm{i} \partial_{\alpha[\beta} A_{\gamma]}{ }^{\delta i j}+\frac{2 \mathrm{i}}{3} \partial_{\beta \gamma} A_{\alpha}{ }^{\delta i j} \\
& +\frac{4 \mathrm{i}}{9} \delta_{[\beta}^{\delta} \partial_{\gamma] \rho} A_{\alpha}{ }^{\rho i j}-\frac{4 \mathrm{i}}{3} \delta_{[\beta}^{\delta} \partial_{|\alpha \rho|} A_{\gamma]}{ }^{\rho i j} \\
& +\frac{4 \mathrm{i}}{9} \delta_{[\beta}^{\delta} \partial_{\gamma] \alpha} A^{i j}+\frac{2 \mathrm{i}}{3} \delta_{\alpha}^{\delta} \partial_{\beta \gamma} A^{i j} \\
& +\varepsilon^{i j} \varepsilon_{\alpha \beta \gamma \rho} \mathcal{S}^{\rho \delta}+\frac{4}{3} \varepsilon^{i j} \delta_{[\beta}^{\delta} \mathcal{A}_{\gamma] \alpha}+2 \varepsilon^{i j} \delta_{\alpha}^{\delta} \mathcal{A}_{\beta \gamma}, \quad \mathcal{S}^{\alpha \beta}=\mathcal{S}^{(\alpha \beta)} .
\end{aligned}
$$

\footnotetext{
${ }^{6}$ In the anomaly-free case, $A^{\alpha i j k}=0$, the component analysis was carried out in [4]. More recently, it was generalised [21] to the case of a special trace supermultiplet $A^{\alpha i j k}$ given by (2.11).

${ }^{7}$ The $\mathrm{SU}(2)$ indices on any field are always assumed to be symmetrized.
} 
Taking successive spinor covariant derivatives of the superfield $J$ satisfying the equation (2.3), one finds the following relations

$$
\begin{aligned}
& D_{\alpha}^{i} J=\Psi_{\alpha}^{i}, \\
& D_{\alpha}^{i} \Psi_{\beta}^{j}=V_{\alpha \beta}^{i j}+\varepsilon^{i j} C_{\alpha \beta}-\mathrm{i} \varepsilon^{i j} \partial_{\alpha \beta} J, \quad C_{\alpha \beta}=C_{(\alpha \beta)}, \quad V_{\alpha \beta}^{i j}=V_{[\alpha \beta]}^{i j}, \\
& D_{\alpha}^{i} C_{\beta \gamma}=\Sigma_{(\beta, \gamma) \alpha}^{i}+\frac{8 \mathrm{i}}{5} \partial_{\alpha(\beta} \Psi_{\gamma)}^{i}, \quad \Sigma_{[\alpha, \gamma \delta]}^{i}=0, \\
& D_{\alpha}^{i} V_{\beta \gamma}{ }^{j k}=\varepsilon^{i(j} \sum_{\alpha, \beta \gamma}^{k)}-\frac{8 \mathrm{i}}{5} \varepsilon^{i(j} \partial_{\alpha[\beta} \Psi_{\gamma]}^{k)}-\frac{2 \mathrm{i}}{5} \varepsilon^{i(j} \partial_{\beta \gamma} \Psi_{\alpha}^{k)}-\varepsilon_{\alpha \beta \gamma \delta} A^{\delta i j k}, \\
& D_{\alpha}^{i} \Sigma_{\beta, \gamma \delta}^{j}=\varepsilon^{i j} \mathcal{T}_{\alpha \beta, \gamma \delta}+\frac{4 \mathrm{i}}{3} \partial_{\alpha \beta} V_{\gamma \delta}^{i j}-\frac{4 \mathrm{i}}{15} \partial_{\gamma \delta} V_{\alpha \beta}{ }^{i j}-\frac{4 \mathrm{i}}{15} \partial_{\beta[\gamma} V_{\delta] \alpha}{ }^{i j}-\frac{4 \mathrm{i}}{3} \partial_{\alpha[\gamma} V_{\delta] \beta}{ }^{i j} \\
& +2 \mathrm{i} \varepsilon^{i j} \partial_{\alpha[\gamma} C_{\delta] \beta}-\frac{2 \mathrm{i}}{5} \varepsilon^{i j} \partial_{\beta[\gamma} C_{\delta] \alpha}+\frac{2 \mathrm{i}}{5} \varepsilon^{i j} \partial_{\gamma \delta} C_{\alpha \beta} \\
& -\varepsilon_{\gamma \delta \epsilon \alpha} A_{\beta}{ }^{\epsilon i j}+\frac{1}{3} \varepsilon_{\gamma \delta \epsilon \beta} A_{\alpha}{ }^{\epsilon i j}, \quad \mathcal{T}_{\alpha[\beta, \gamma \delta]}=0, \quad \mathcal{T}_{\alpha \beta, \gamma \delta}=\mathcal{T}_{[\alpha \beta],[\gamma \delta]}, \\
& D_{\epsilon}^{i} \mathcal{T}_{\alpha \beta, \gamma \delta}=\frac{2 \mathrm{i}}{3} \partial_{[\alpha[\gamma} \Sigma_{|\epsilon|, \delta] \beta]}^{i}+2 \mathrm{i} \partial_{\epsilon[\alpha} \Sigma_{\beta], \gamma \delta}^{i}+2 \mathrm{i} \partial_{\epsilon[\gamma} \Sigma_{\delta], \alpha \beta}^{i} \\
& +\frac{\mathrm{i}}{3} \partial_{\alpha \beta} \Sigma_{\epsilon, \gamma \delta}^{i}+\frac{\mathrm{i}}{3} \partial_{\gamma \delta} \Sigma_{\epsilon, \alpha \beta}^{i}+\frac{1}{2} \varepsilon_{\alpha \beta \rho \epsilon} \Lambda_{\gamma \delta}{ }^{\rho i}+\frac{1}{2} \varepsilon_{\gamma \delta \rho \epsilon} \Lambda_{\alpha \beta}{ }^{\rho i},
\end{aligned}
$$

as well as the conditions

$$
\partial^{\alpha \beta} V_{\alpha \beta}{ }^{i j}=4 \mathrm{i} A^{i j}, \quad \partial^{\alpha \beta} \Sigma_{\gamma, \alpha \beta}^{i}=4 \mathrm{i} \Lambda_{\gamma}^{i}, \quad \partial^{\alpha \beta} \mathcal{T}_{\alpha \beta, \gamma \delta}=4 \mathrm{i} \mathcal{A}_{\gamma \delta}
$$

Note that the algebraic properties of the tensor $\mathcal{T}_{\alpha \beta, \gamma \delta}$, which are given in eq. (2.8e), imply the identity

$$
\mathcal{T}_{\alpha \beta, \gamma \delta}=\mathcal{T}_{\gamma \delta, \alpha \beta}
$$

As a result, if we convert each of the two pairs of spinor indices of $\mathcal{T}_{\alpha \beta, \gamma \delta}$ into vector ones by the standard rule $V_{\alpha \beta}=-V_{\beta \alpha} \rightarrow V_{a}=\frac{1}{4}\left(\tilde{\gamma}_{a}\right)^{\alpha \beta} V_{\alpha \beta}$, we end up with a second-rank tensor $\mathcal{T}_{a b}$, which is symmetric and traceless, $\mathcal{T}_{a b}=\mathcal{T}_{b a}$ and $\mathcal{T}^{a}{ }_{a}=0$.

The equations (2.9) tell us that if $A^{\alpha i j k}=0$ the component projections of $V_{\alpha \beta}{ }^{i j}, \Sigma_{\gamma, \alpha \beta}^{i}$ and $\mathcal{T}_{\alpha \beta, \gamma \delta}$ are proportional to the conserved $\mathrm{SU}(2)$ current, supersymmetry current and energy-momentum tensor, respectively. ${ }^{8}$ If an arbitrary trace superfied $A^{\alpha i j k}$ is switched on, they are no longer conserved. It follows from (2.9) that in order to be able to specify a conserved supersymmetry current it is necessary to require that $\Lambda_{\alpha}^{i}$ is a vector divergence, $\Lambda_{\alpha}^{i}=\partial^{b} \tilde{\Sigma}_{\alpha, b}^{i}$. It is now important to note that if $\Lambda_{\alpha}^{i}$ is a divergence then so is $\mathcal{A}_{\alpha \beta}$, i.e. a conserved supersymmetry current automatically implies a conserved energy-momentum tensor. Similarly, a conserved SU(2) current implies both a conserved supercurrent and a conserved energy-momentum tensor. One should, however, keep in mind that the conserved supersymmetry current and energy-momentum tensor need no longer be $\gamma$-traceless and traceless, respectively.

Let us see how this works for the non-conformal supercurrent involving an $\mathcal{O}(4)$ multiplet [21]. The trace superfield $A^{\alpha i j k}$ is chosen to be proportional to

$$
A^{\alpha i j k}=\mathrm{i} \partial^{\alpha \beta} D_{\beta l} \mathbb{L}^{i j k l},
$$

\footnotetext{
${ }^{8}$ One can also verify that the supercurrent has $40+40$ component degrees of freedom.
} 
where $\mathbb{L}^{i j k l}=\mathbb{L}^{(i j k l)}$ satisfies the reality condition $\overline{\mathbb{L}^{i j k l}}=\mathbb{L}_{i j k l}$ and the defining constraint for an $\mathcal{O}(4)$ multiplet

$$
D_{\alpha}^{(i} \mathbb{L}^{j k l p)}=0 .
$$

It is simple to check that this superfield satisfies the integrability condition (2.5) in the flat case. Furthermore, since the trace multiplet (2.11) is a divergence, its descendent $A^{i j}$ is a divergence and a conserved $\mathrm{SU}(2)$ current can be introduced. As remarked above, it then follows that a conserved $Q$-supersymmetry current and energy-momentum tensor exist as well. These currents may be defined as follows:

$$
\begin{aligned}
j_{\alpha \beta}{ }^{i j} & =V_{\alpha \beta}{ }^{i j}+\frac{3}{4} D_{\alpha k} D_{\beta l} \mathbb{L}^{i j k l}, & \partial^{\alpha \beta} j_{\alpha \beta}{ }^{i j} & =0, \\
S_{\alpha \beta, \gamma}{ }^{i} & =\Sigma_{\gamma, \alpha \beta}^{i}+\frac{1}{2} D_{\gamma j} D_{[\alpha k} D_{\beta] l} \mathbb{L}^{i j k l}, & \partial^{\alpha \beta} S_{\alpha \beta, \gamma}{ }^{i} & =0, \\
T_{\alpha \beta, \gamma \delta} & =\mathcal{T}_{\alpha \beta, \gamma \delta}+\frac{1}{4} D_{[\alpha i} D_{\beta j} D_{\gamma k} D_{\delta]} \mathbb{L}^{i j k l}, & & \partial^{\alpha \beta} T_{\alpha \beta, \gamma \delta}=0 .
\end{aligned}
$$

Note that neither is the $Q$-supersymmetry current $\gamma$-traceless nor is the energy-momentum tensor traceless [21].

Within the conformal approach, the form of the trace superfield $A_{\alpha}{ }^{i j k}$ should depend on conformal compensators. Therefore, a natural question one can ask is: what compensator(s) should one associate with the construction (2.11)? Furthermore, how do we lift the construction (2.11) to a primary superfield with the use of a compensator in conformal supergravity? One can show that if we assume that the compensator is a tensor multiplet and we try to lift the construction (2.11) to conformal superspace (see appendix A), it is not possible to add compensator dependant terms such that the condition (2.5) is satisfied. On the other hand, if the compensator was an $\mathcal{O}(2)$ (or linear) multiplet one would expect a symmetric $\mathrm{SU}(2)$ tensor to appear in the construction $(2.11)$, which is not the case. ${ }^{9}$ For this reason, it is necessary to use a different scalar compensating superfield instead that of the tensor multiplet. We will present the appropriate compensator and show how to generalise the construction in [21] to supergravity in section 2.3 .

It is elucidating to ask what can be learned by allowing the $\mathcal{O}(4)$ multiplet $\mathbb{L}^{i j k l}$ to be composite. For instance, suppose we have two $\mathcal{O}(2)$ multiplets described by the superfields $G^{i j}=G^{(i j)}$ and $H^{i j}=H^{(i j)}$, which satisfy the differential constraints

$$
D_{\alpha}^{(i} G^{j k)}=D_{\alpha}^{(i} H^{j k)}=0 .
$$

We can then construct

$$
\mathbb{L}^{i j k l}=G^{(i j} H^{k l)}
$$

We will further assume $G^{i j}$ has a nowhere vanishing magnitude $G \neq 0$, which is defined by $G^{2}:=\frac{1}{2} G^{i j} G_{i j}$. If we freeze $G$ to a constant we find

$$
0=D_{\alpha}^{i} G^{2}=\frac{2}{3} G^{i j} D_{\alpha}^{k} G_{j k} \quad \Longrightarrow \quad D_{\alpha}^{i} G^{j k}=\frac{2}{3} \varepsilon^{i(j} D_{\alpha k} G^{j) k}=0,
$$

\footnotetext{
${ }^{9}$ The SU(2) tensor corresponding to the superfield describing the $\mathcal{O}(2)$ multiplet can be set to a constant using super-Weyl transformations.
} 
where we used $G^{i j} G_{j k}=\delta_{k}^{i} G^{2}$. Using (2.16), the superfield $A^{\alpha i j k}$ can be seen to take the form

$$
A^{\alpha i j k}=\mathrm{i} G^{(i j} \mathbb{W}^{\alpha k)},
$$

where we have defined

$$
\mathbb{W}^{\alpha i}:=\frac{5}{6} \partial^{\alpha \beta} D_{\beta j} H^{i j} .
$$

One can verify that $\mathbb{W}^{\alpha i}$ satisfies the following differential constraints:

$$
\begin{aligned}
D_{\alpha}^{(i} \mathbb{W}^{\beta j)} & =\frac{1}{4} \delta_{\alpha}^{\beta} D_{\gamma}^{(i} \mathbb{W}^{\gamma j)}, \\
D_{\alpha i} \mathbb{W}^{\alpha i} & =0,
\end{aligned}
$$

which correspond to those of a vector multiplet, see e.g. appendix C.

It is important to point out that the representation (2.17) actually implies the existence of a conserved supersymmetry current and energy-momentum tensor due to the constraints (2.19a) and (2.19b), irrespective of the form (2.18). In other words, we only need to require $\mathbb{W}^{\alpha i}$ to be an off-shell vector multiplet for these currents to exist. In particular, the constraint (2.19a) implies the condition (2.5), while the constraint (2.19b) is required to show ${ }^{10}$

$$
\Lambda_{\alpha}^{i}=\frac{1}{8} D_{\alpha j} D_{\beta k} A^{\beta i j k}=\frac{2}{3} \partial_{\alpha \beta}\left(G^{i j} \mathbb{W}_{j}^{\beta}\right),
$$

which ensures the existence of a conserved supersymmetry current that is, however, no longer $\gamma$-traceless, as well as a conserved energy-momentum tensor that is no longer traceless. These may be defined as

$$
\begin{aligned}
S_{\alpha \beta, \gamma}{ }^{i} & :=\Sigma_{\gamma, \alpha \beta}^{i}-\frac{4 \mathrm{i}}{3} \varepsilon_{\alpha \beta \gamma \delta} G^{i j} \mathbb{W}_{j}^{\delta}, \quad \partial^{\alpha \beta} S_{\alpha \beta, \gamma}{ }^{i}=0, \\
T_{\alpha \beta, \gamma \delta} & :=\mathcal{T}_{\alpha \beta, \gamma \delta}+\frac{\mathrm{i}}{6} \varepsilon_{\alpha \beta \gamma \delta} G^{i j} D_{\epsilon i} \mathbb{W}_{j}^{\epsilon}, \quad \partial^{\alpha \beta} T_{\alpha \beta, \gamma \delta}=0 .
\end{aligned}
$$

The off-shell conditions (2.19a) and (2.19b) do not lead to an SU(2) current, since one finds

$$
A^{i j}=\frac{\mathrm{i}}{8} G^{k(i} D_{\alpha}^{j)} \mathbb{W}_{k}^{\alpha}
$$

However, as $G^{i j} A_{i j}=0$ we can instead introduce a conserved $\mathrm{U}(1)$ current $j_{\alpha \beta}$ defined by

$$
j_{\alpha \beta}=V_{\alpha \beta}{ }^{i j} G_{i j}, \quad \partial^{\alpha \beta} j_{\alpha \beta}=0,
$$

where the $\mathrm{U}(1)$ subgroup is the stability group of $G^{i j}$. Only when the equations of motion require $D_{\alpha}^{(i} \mathbb{W}^{\alpha j)}$ to be a vector divergence up to terms proportional to $G^{i j}$ can a conserved $\mathrm{SU}(2)$ current be introduced.

We conclude this section by emphasising once more that eq. (2.17) leads to a nonconformal supercurrent for any vector multiplet $\mathbb{W}^{\alpha i}$. The appearance of the constant $\mathrm{SU}(2)$ tensor $G^{i j}$ is to be interpreted as a compensator that has been frozen. It is precisely the form (2.17) that we will generalise to curved superspace in the next subsection and it will be verified by further analysis and a worked example.

\footnotetext{
${ }^{10}$ Keep in mind that $G^{i j}$ is constant.
} 


\subsection{The non-conformal supercurrent based on a compensating $\mathcal{O}(2)$ multiplet}

Let us first describe how the conservation condition on the supercurrent originates from superspace. We will consider a supergravity action with a compensating $\mathcal{O}(2)$ multiplet $G^{i j}$. The supergravity equations of motion in superspace can be easily obtained if one knows the dependence of the supergravity action on the unconstrained superfield prepotential for $\mathcal{N}=(1,0)$ conformal supergravity. It is a real primary scalar $H$ [4] of dimension -2 with supergravity gauge transformation ${ }^{11}$

$$
\delta H=\nabla^{\alpha i j k} \Lambda_{\alpha i j k}, \quad \Lambda_{\alpha i j k}=\Lambda_{\alpha(i j k)} .
$$

In general, the constrained superfields must also transform under such a gauge transformation since their constraints must be preserved under shifts in the supergravity prepotential. This tells us that under the gauge transformation (2.24) the prepotential for the $\mathcal{O}(2)$ multiplet $\rho_{\alpha i}$ should transform. For a description of the $\mathcal{O}(2)$ multiplet in terms of its prepotential in supergravity see appendix B.

If any additional matter fields other than the compensator are chosen to obey their equations of motion, a general variation of the action with respect to the supergravity and compensator prepotentials becomes

$$
\delta S=\int \mathrm{d}^{6 \mid 8} z E\left(\delta H J+\delta \rho_{\alpha}^{i} \mathbb{W}_{i}^{\alpha}\right) .
$$

The prepotential $\rho_{\alpha}^{i}$ is defined modulo gauge transformations

$$
\rho_{\alpha}^{i} \rightarrow \rho_{\alpha}^{\prime i}=\rho_{\alpha}^{i}+\nabla_{\alpha}^{i} \tau+\nabla_{\beta j} \tau_{\alpha}{ }^{\beta i j}, \quad \tau_{\alpha}{ }^{\alpha i j}=0, \quad \tau_{\alpha}{ }^{\beta i j}=\tau_{\alpha}{ }^{\beta(i j)},
$$

where $\tau$ and $\tau_{\alpha}{ }^{\beta i j}$ are dimensionless primary superfields. In order for the action to be invariant under these gauge transformations, the superfield $\mathbb{W}_{i}^{\alpha}$ must obey the constraints

$$
\nabla_{\alpha}^{(i} \mathbb{W}^{\beta j)}=\frac{1}{4} \delta_{\alpha}^{\beta} \nabla_{\gamma}^{(i} \mathbb{W}^{\gamma j)}, \quad \nabla_{\alpha i} \mathbb{W}^{\alpha i}=0,
$$

which are characteristic of the field strength of a vector multiplet. Now we wish to specialise to the supergravity gauge transformations where $\delta S=0$ but we need to know the transformation of the prepotential $\rho_{\alpha i}$. Its transformation should involve the supergravity gauge parameter $\Lambda_{\alpha i j k}$ and covariant fields of the compensating $\mathcal{O}(2)$ multiplet since we should obtain a covariant conservation equation. ${ }^{12}$ On dimensional grounds we must have ${ }^{13}$

$$
\delta \rho_{\alpha i}=\mathrm{i} \Lambda_{\alpha i j k} G^{j k} .
$$

Requiring $\delta S=0$ under the supergravity gauge transformations leads to the non-conformal conservation equation

$$
\nabla^{\alpha i j k} J=\mathrm{i} G^{(i j} \mathbb{W}^{\alpha k)},
$$

\footnotetext{
${ }^{11}$ The gauge transformation presented here is the unique extension of the linearised transformation in [4] to curved superspace, assuming the gauge parameter $\Lambda_{\alpha i j k}$ is primary.

${ }^{12}$ The transformation must also be linear in the fields of the $\mathcal{O}(2)$ multiplet since its prepotential description must remain unchanged. We will also verify this by an explicit example.

${ }^{13}$ We can always rescale $G^{i j}$ by fixing its relative normalisation to its prepotential.
} 
where the $\mathcal{O}(2)$ multiplet in conformal superspace is a primary superfield $G^{i j}=G^{(i j)}$ of dimension 4 satisfying the constraint

$$
\nabla_{\alpha}^{(i} G^{j k)}=0
$$

Therefore, the general form of the trace superfield $A^{\alpha i j k}$ in the presence of a compensating nowhere vanishing $\mathcal{O}(2)$ multiplet (with $G:=\frac{1}{2} G^{i j} G_{i j} \neq 0$ ) in supergravity is

$$
A^{\alpha i j k}=\mathrm{i} G^{(i j} \mathbb{W}^{\alpha k)},
$$

where $\mathbb{W}^{\alpha i}$ is the composite vector multiplet determined by the theory via (2.25).

It is interesting to note that if we weaken the constraint defining the $\mathcal{O}(2)$ multiplet to a deformed $\mathcal{O}(2)$ multiplet $[42,43]$

$$
\nabla_{\alpha}^{(i} G^{j k)}=\mathrm{i} \varepsilon_{\alpha \beta \gamma \delta} \mathbb{W}^{\beta(i} \mathbb{W}^{\gamma j} \mathbb{W}^{\delta k)},
$$

the postulated trace superfield (2.31) still satisfies the consistency condition (2.5) but a conserved supersymmetry current is no longer guaranteed.

\subsubsection{An example: Abelian gauge theory coupled to an $\mathcal{O}(2)$ multiplet}

We now provide an explicit example of a non-conformal supercurrent in curved superspace. Consider an Abelian gauge theory coupled to a linear multiplet. The action for the theory is built out of two supersymmetric invariants: (i) a higher-derivative Abelian vector multiplet action; and (ii) a $B F$ action giving rise to the coupling of the vector multiplet to the linear (or $\mathcal{O}(2))$ multiplet.

A supersymmetric $F \square F$ action was described in [44] in Minkowski superspace and in conformal supergravity in [41]. It is straightforward to construct its supercurrent (up to some normalisation). In the Abelian case, it is ${ }^{14}$

$$
J=\frac{3}{8} X^{i j} X_{i j}+\frac{\mathrm{i}}{2} W^{\alpha i} \nabla_{\alpha \beta} W_{i}^{\beta}+\frac{1}{4} F_{\alpha}^{\beta} F_{\beta}{ }^{\alpha},
$$

while the equation of motion for the vector multiplet is

$$
\mathcal{G}^{i j}=0, \quad \mathcal{G}^{i j}:=\square X^{i j}-2 Y_{\alpha}{ }^{\beta i j} F_{\beta}{ }^{\alpha}+\frac{5}{2} X^{\alpha(i} \overleftrightarrow{\nabla}_{\alpha \beta} W^{\beta j)},
$$

where we have defined $\square:=\nabla^{a} \nabla_{a}$ and $S \overleftrightarrow{\nabla}_{a} U:=S \nabla_{a} U-\left(\nabla_{a} S\right) U$ for arbitrary superfields $S$ and $U$. One can check that, upon using the equations of motion, the supercurrent is conserved

$$
\nabla^{\alpha i j k} J=\mathrm{i} W^{\alpha(i} \mathcal{G}^{j k)}=0 .
$$

The non-conformal conservation condition can be deduced if we consider the $F \square F$ action coupled to a $B F$ action, where $B$ is the gauge four-form of a nowhere vanishing $\mathcal{O}(2)$ multiplet. The $B F$ action is just the action formula for the product of a vector and an $\mathcal{O}(2)$ multiplet $[45,46]$,

$$
S_{B F}=\int \mathrm{d}^{6 \mid 8} z E W^{\alpha i} \rho_{\alpha i}
$$

\footnotetext{
${ }^{14}$ We refer the reader to appendix $\mathrm{C}$ for our notation and conventions regarding the vector multiplet.
} 
We take the $\mathcal{O}(2)$ multiplet as a conformal compensator. For the combined action incorporating the $F \square F$ action and the $B F$ action, the equation of motion for the vector multiplet becomes

$$
\mathcal{G}^{i j}=\lambda G^{i j}
$$

where $\lambda$ is some constant and $G^{i j}$ is the compensating $\mathcal{O}(2)$ multiplet with prepotential $\rho_{\alpha i}$. It is straightforward to check that the non-conformal conservation equation (2.29) holds due to the equation of motion,

$$
\nabla^{\alpha i j k} J=\mathrm{i} G^{(i j} \mathbb{W}^{\alpha k)}, \quad \mathbb{W}^{\alpha i}=\lambda W^{\alpha i},
$$

which verifies the supercurrent conservation equation for a compensating $\mathcal{O}(2)$ multiplet. In the above computation it is important to note that the supercurrent for the combined action does not obtain a contribution from the $B F$ action since it does not depend on the supergravity prepotential as it admits a topological realisation.

Finally, it is worth mentioning that the equations of motion for this example lead to a conserved SU(2) current. Indeed, using (2.37) and (2.34) we find, after reducing to flat superspace,

$$
A^{i j}=\frac{\lambda}{2} G^{k(i} X_{k}^{j)}=\frac{1}{2}\left(\square X^{k(i}\right) X_{k}^{j)}=\frac{1}{2} \partial^{a}\left(\left(\partial_{a} X^{k(i}\right) X_{k}^{j)}\right) .
$$

The conserved $\mathrm{SU}(2)$ current is therefore

$$
j_{\alpha \beta}{ }^{i j}=V_{\alpha \beta}{ }^{i j}-\frac{\mathrm{i}}{2}\left(\partial_{\alpha \beta} X^{k(i}\right) X_{k}^{j)}, \quad \partial^{\alpha \beta} j_{\alpha \beta}{ }^{i j}=0 .
$$

\subsection{The non-conformal supercurrent involving an $\mathcal{O}(4)$ multiplet}

The trace multiplet based on the $\mathcal{O}(4)$ multiplet, eq. (2.11), must correspond to a conformal compensator described by a scalar superfield which cannot be the tensor multiplet as mentioned previously. It turns out that the right compensating multiplet is built from a primary dimension -4 scalar superfield $T$ subject to the constraint ${ }^{15}$

$$
\nabla_{(\alpha}^{k} \nabla_{\beta) k} T=0 \Longrightarrow \nabla_{(\alpha}^{i} \nabla_{\beta)}^{j} T=0 .
$$

It corresponds to the $\mathcal{O}^{*}(4)$ multiplet described in [49] and the above constraint can be solved in terms of an unconstrained prepotential $U_{i j k l}=U_{(i j k l)}$ as $^{16}$

$$
T=\nabla^{i j k l} U_{i j k l}, \quad \nabla^{i j k l}:=\frac{1}{4 !} \varepsilon^{\alpha \beta \gamma \delta} \nabla_{\alpha}^{(i} \nabla_{\beta}^{j} \nabla_{\gamma}^{k} \nabla_{\delta}^{l)} .
$$

Here $U_{i j k l}$ is primary and of dimension -6 . One can check that $U_{i j k l}$ is defined up to the gauge transformations

$$
\delta U_{i j k l}=\nabla_{\alpha}^{m} \xi^{\alpha}{ }_{i j k l m}
$$

where $\xi^{\alpha}{ }_{i j k l m}=\xi^{\alpha}{ }_{(i j k l m)}$.

\footnotetext{
${ }^{15}$ Coupling this multiplet to conformal supergravity is equivalent to working in the $\mathrm{SU}(2)$ superspace formulation of [36] and setting the torsion component $N_{\alpha \beta}=0$.

${ }^{16}$ This was first worked out in Minkowski superspace in [4].
} 
We wish to work out the supercurrent conservation equation in the presence of the compensating $\mathcal{O}^{*}(4)$ multiplet. Let us first consider the general variation of the action with respect to the supergravity and matter prepotentials

$$
\delta S=\int \mathrm{d}^{6 \mid 8} z E\left(\delta H J+\delta U_{i j k l} \mathbb{L}^{i j k l}\right),
$$

where $H$ is the superfield prepotential for conformal supergravity and $\mathbb{L}^{i j k l}$ is some superfield of dimension 8 required to be an $\mathcal{O}(4)$ multiplet as a result of the gauge transformation law (2.43). As in the previous subsection, in the variation (2.44) we have assumed any additional matter fields satisfy their equations of motion. The conformal supergravity prepotential transforms under the supergravity gauge transformations as eq. (2.24) and $\delta U_{i j k l}$ should be expressed in terms of three spinor derivatives hitting $\Lambda_{\alpha i j k}$ on dimensional grounds. In any case, this should lead to a trace superfield $A^{\alpha i j k}$ linear in both the $\mathcal{O}^{*}(4)$ multiplet $T$ and the $\mathcal{O}(4)$ multiplet $\mathbb{L}^{i j k l}$.

We expect that $A^{\alpha i j k}$, reduces to the construction in [21] after fixing $T$ to a constant and reducing to flat superspace. Taking this into account and considering all possible terms linear in $T$ and $\mathbb{L}^{i j k l}$ in conformal superspace, one can construct the most general ansatz for the trace superfield. Then demanding the consistency condition (2.5) fixes it as

$$
\begin{aligned}
A^{\alpha i j k}= & \mathrm{i} T \nabla^{\alpha \beta} \nabla_{\beta l} \mathbb{L}^{i j k l}+\frac{3}{16} \varepsilon^{\alpha \beta \gamma \delta}\left(\nabla_{\beta}^{(i} T\right) \nabla_{\gamma l} \nabla_{\delta p} \mathbb{L}^{j k) l p}+\frac{15 \mathrm{i}}{4}\left(\nabla_{\beta l} T\right) \nabla^{\alpha \beta} \mathbb{L}^{i j k l} \\
& +\frac{3}{4} \varepsilon^{\alpha \beta \gamma \delta}\left(\nabla_{\gamma}^{(i} \nabla_{\delta l} T\right) \nabla_{\beta p} \mathbb{L}^{j k) l p}+\frac{5 \mathrm{i}}{2}\left(\nabla^{\alpha \beta} \nabla_{\beta l} T\right) \mathbb{L}^{i j k l}+\frac{5}{8} \varepsilon^{\alpha \beta \gamma \delta}\left(\nabla_{\beta}^{(i} \nabla_{\gamma l} \nabla_{\delta p} T\right) \mathbb{L}^{j k) l p} \\
& -25 X_{l}^{\alpha} T \mathbb{L}^{i j k l}+4 \mathrm{i} W^{\alpha \beta} T \nabla_{\beta l} \mathbb{L}^{i j k l}+15 \mathrm{i} W^{\alpha \beta}\left(\nabla_{\beta l} T\right) \mathbb{L}^{i j k l} .
\end{aligned}
$$

One can check that this is also primary. If we gauge fix $T=1$ and reduce to Minkowski superspace, $\nabla_{A} \rightarrow\left(\partial_{a}, D_{\alpha}^{i}\right)$ and $W^{\alpha \beta} \rightarrow 0$, we obviously recover (2.11).

The existence of conserved $\mathrm{SU}(2)$ and supersymmetry currents is then guaranteed by the results (2.13).

\subsubsection{An example: the relaxed hypermultiplet}

It is illustrative to provide an example of a non-conformal supercurrent in curved superspace with $T$ chosen as a compensating superfield.

In the case of $4 \mathrm{D} \mathcal{N}=2$ Poincare supersymmetry, the relaxed hypermultiplet [47] was the first off-shell formulation without intrinsic central charge for the massless hypermultiplet. This formulation was generalised to $6 \mathrm{D} \mathcal{N}=(1,0)$ supersymmetry in [4]. In both cases, the relaxed hypermultiplet was described only in Minkowski superspace. To the best of our knowledge, its coupling to supergravity has never been constructed. Such a coupling will be given below. In conformal supergravity one must introduce a compensating $\mathcal{O}^{*}(4)$ multiplet as we will show.

To begin with, the relaxed hypermultiplet is described by the superfields $L^{i j}, L^{i j k l}$ and $\tilde{T}$, subject to the following off-shell constraints

$$
\begin{aligned}
\nabla_{\alpha}^{(i} L^{j k)} & =T \nabla_{\alpha l} L^{i j k l}+5\left(\nabla_{\alpha l} T\right) L^{i j k l}, \\
\nabla_{\alpha}^{(i} L^{j k l p)} & =0, \\
\nabla_{(\alpha}^{j} \nabla_{\beta) j} \tilde{T} & =0 .
\end{aligned}
$$


The independent off-shell component fields of the relaxed hypermultiplet can be extracted from the above constraints.

The action for the relaxed hypermultiplet may be described in a covariant way using the primary superform action $[41,48]$, which is built out of a primary superfield $A_{\alpha}{ }^{i j k} .{ }^{17}$ It satisfies the differential constraint

$$
\nabla_{(\alpha}^{(i} A_{\beta}{ }^{j k l)}=0
$$

One only needs to allow the superfield $A_{\alpha}{ }^{i j k}$, taking on the role of a Langrangian, to be composed of the fields of the relaxed hypermultiplet and the compensating superfield $T$. The two supersymmetry invariants making up the action for the relaxed hypermultiplet are: (i) $I_{1}$ described by

$$
A_{\alpha}^{i j k}=T \nabla_{\alpha l} \mathbb{H}^{i j k l}+5\left(\nabla_{\alpha l} T\right) \mathbb{H}^{i j k l}
$$

where

$$
\mathbb{H}^{i j k l}=\frac{2}{5} L^{(i j} L^{k l)}-\frac{4}{3} T L_{p}^{(i} L^{j k l) p}-\frac{15}{7} T^{2} L^{m n(i j} L^{k l)}{ }_{m n}
$$

and (ii) $I_{2}$ described by

$$
A_{\alpha}^{i j k}=\tilde{T} \nabla_{\alpha l} L^{i j k l}+5\left(\nabla_{\alpha l} \tilde{T}\right) L^{i j k l} .
$$

Their linear combination gives the relaxed hypermultiplet action. ${ }^{18}$

The superspace equations of motion for the relaxed hypermultiplet action are

$$
T \nabla_{\alpha j} L^{i j}+3\left(\nabla_{\alpha j} T\right) L^{i j}=\lambda \nabla_{\alpha}^{i}\left(\frac{\tilde{T}}{T}\right), \quad L^{i j k l}=0,
$$

where $\lambda$ is some non-zero constant related to the relative coefficients of the invariants. The equations of motion (2.51) are constructed such that they are primary and that they reduce to those given in Minkowski superspace in [47] when $T$ is set to a constant. Note that, up to a constant, the equations of motion completely determine $\tilde{T}$ in terms of other fields. The supercurrent is

$$
J=T L^{i j} L_{i j},
$$

which is the unique primary scalar that is linear in $T$, quadratic in $L^{i j}$ and of dimension 4.

One may verify that the supercurrent conservation equation (2.3) recovers (2.45) with a composite multiplet

$$
\mathbb{L}^{i j k l}=-\frac{4}{5} L^{(i j} L^{k l)}
$$

which is an $\mathcal{O}(4)$ multiplet once one imposes the equations of motion for the relaxed hypermultiplet. This provides an example verifying the supercurrent conservation equation for a compensating $\mathcal{O}^{*}(4)$ multiplet.

\footnotetext{
${ }^{17}$ The superfield $A_{\alpha}{ }^{i j k}$ should not be confused with $A^{\alpha i j k}$.

${ }^{18}$ Note that one could also choose $T=G^{-1}$ since $G^{-1}$ satisfies the appropriate differential constraint.
} 


\subsection{Further generalisations}

So far we have found two solutions for the trace superfield $A^{\alpha i j k}$ which lead to a conserved supercurrent and energy-momentum tensor, cf. subsections 2.2 and 2.3. One involves an $\mathcal{O}(2)$ multiplet with a vector multiplet, while the other involves an $\mathcal{O}(4)$ multiplet with an $\mathcal{O}^{*}(4)$ multiplet. It turns out there is in an infinite family of solutions that involve the product of an $\mathcal{O}(n)$ multiplet with an $\mathcal{O}^{*}(n)$ multiplet for $n \geq 2 .{ }^{19}$ The $\mathcal{O}^{*}(n)$ multiplets were introduced in [49] as 'dual' to the $\mathcal{O}(n)$ multiplets in the sense that there exists an action formula that schematically involves the product of the two. We will describe the defining constraints of these multiplets below and introduce the infinite family of non-conformal supercurrents.

The $\mathcal{O}(n)$ multiplet for $n \geq 1$ is given by a primary superfield $L^{i_{1} \cdots i_{n}}$ of dimension $2 n$ satisfying the differential constraint

$$
\nabla_{\alpha}^{\left(i_{1}\right.} L^{\left.i_{2} \cdots i_{n+1}\right)}=0
$$

They are off-shell for $n \geq 2$.

The $\mathcal{O}^{*}(3)$ multiplet is described by a primary superfield $T_{\alpha}$ of dimension $-3 / 2$ with the differential constraint

$$
\nabla_{(\alpha}^{i} T_{\beta)}=0
$$

while the $\mathcal{O}^{*}(n)$ multiplet with $n>4$ is described by a superfield $T_{i_{1} \cdots i_{n-4}}$ of dimension $4-2 n$ satisfying the constraint

$$
\nabla_{\alpha}^{j} T_{i_{1} \cdots i_{n-5} j}=0 \quad \Longrightarrow \quad \nabla_{(\alpha}^{j} \nabla_{\beta) j} T_{i_{1} \cdots i_{n-4}}=0
$$

The prepotential formulations for these multiplets appeared in [49].

One can build a primary superfield $A^{\alpha i j k}$ satisfying (2.5) out of an $\mathcal{O}(3)$ multiplet and an $\mathcal{O}^{*}(3)$ multiplet as follows

$$
\begin{aligned}
A^{\alpha i j k}= & T_{\beta} \nabla^{\alpha \beta} \mathbb{L}^{i j k}-\frac{3 \mathrm{i}}{16} \varepsilon^{\alpha \beta \gamma \delta}\left(\nabla_{\beta}^{(i} T_{\gamma}\right) \nabla_{\delta l} \mathbb{L}^{j k) l}-\frac{\mathrm{i}}{4} \varepsilon^{\alpha \beta \gamma \delta}\left(\nabla_{\beta}^{(i} \nabla_{\gamma l} T_{\delta}\right) L^{j k) l}+\left(\nabla^{\alpha \beta} T_{\beta}\right) \mathbb{L}^{i j k} \\
& +6 W^{\alpha \beta} T_{\beta} \mathbb{L}^{i j k} .
\end{aligned}
$$

\footnotetext{
${ }^{19}$ The $\mathcal{O}^{*}(2)$ multiplet is defined to be a vector multiplet [49].
} 
One can do the same with an $\mathcal{O}(4+p)$ and an $\mathcal{O}^{*}(4+p)$ multiplet with $p>0$ as follows

$$
\begin{aligned}
A^{\alpha i j k}= & \frac{p}{2(p+4)} T_{i_{1} \cdots i_{p-1}}{ }^{(i} \nabla_{l m n}^{\alpha} \mathbb{L}^{j k) i_{1} \cdots i_{p-1} l m n}+\mathrm{i} T_{i_{1} \cdots i_{p}} \nabla^{\alpha \beta} \nabla_{\beta l} \mathbb{L}^{i j k l i_{1} \cdots i_{p}} \\
& +\frac{p+3}{2(p+4)}\left(\nabla_{\beta}^{(i} T_{i_{1} \cdots i_{p}}\right) \nabla_{l m}^{\alpha \beta} \mathbb{L}^{j k) l m i_{1} \cdots i_{p}}+\frac{\mathrm{i}(p+3)(p+5)}{(p+1)(p+4)} \nabla_{\beta i_{1}} T_{i_{2} \cdots i_{p+1}} \nabla^{\alpha \beta} \mathbb{L}^{i j k i_{1} \cdots i_{p+1}} \\
& +\frac{p+3}{4(p+1)} \varepsilon^{\alpha \beta \gamma \delta}\left(\nabla_{\gamma}^{(i} \nabla_{\delta i_{1}} T_{i_{2} \cdots i_{p+1}}\right) \nabla_{\beta l} \mathbb{L}^{j k) l i_{1} \cdots i_{p+1}} \\
& +\frac{(p+3)(p+5)}{12(p+2)(p+1)} \varepsilon^{\alpha \beta \gamma \delta}\left(\nabla_{\beta}^{(i} \nabla_{\gamma i_{1}} \nabla_{\delta i_{2}} T_{i_{3} \cdots i_{p+2}}\right) \mathbb{L}^{j k) i_{1} \cdots i_{p+2}} \\
& +\frac{\mathrm{i}(p+3)(p+5)}{3(p+1)(p+2)}\left(\nabla^{\alpha \beta} \nabla_{\beta i_{1}} T_{i_{2} \cdots i_{p+1}}\right) \mathbb{L}^{i j k i_{1} \cdots i_{p+1}} \\
& -\frac{10(p+1)(p+5)}{p+2} X_{i_{1}}^{\alpha} T_{i_{2} \cdots i_{p+1}} \mathbb{L}^{i j k i_{1} \cdots i_{p+1}}+4 \mathrm{i} W^{\alpha \beta} T_{i_{1} \cdots i_{p}} \nabla_{\beta l} \mathbb{L}^{i j k l i_{1} \cdots i_{p}} \\
& +\frac{2 \mathrm{i}(p+3)(p+5)}{(p+1)(p+2)} W^{\alpha \beta}\left(\nabla_{\beta i_{1}} T_{i_{2} \cdots i_{p+1}}\right) \mathbb{L}^{i j k i_{1} \cdots i_{p+1}},
\end{aligned}
$$

where we have introduced the definition

$$
\nabla^{\alpha \beta i j}:=\frac{1}{2} \varepsilon^{\alpha \beta \gamma \delta} \nabla_{\gamma}^{(i} \nabla_{\delta}^{j)}
$$

One can check that when $p=0$, the non-conformal supercurrent corresponding to (2.58) agrees with (2.45). The above general form for the trace superfield corresponds to compensating $\mathcal{O}^{*}(n)$ multiplets. However, for $n \geq 4$ the general form also makes sense if one takes the conformal compensator to be an $\mathcal{O}(n)$ multiplet. It can be checked that in either case, after freezing the compensator to a constant and reducing to flat superspace, one obtains a conserved $\mathrm{SU}(2)$ and, therefore, also a conserved supersymmetry current and energy-momentum tensor. Higher-derivative actions for both cases were described in [49].

\section{The supercurrent associated with the dilaton-Weyl multiplet}

As mentioned earlier, the tensor multiplet may be used as a conformal compensator in supergravity [46]. In conformal superspace, the tensor multiplet is described by a primary superfield $\Phi$ of dimension 2 satisfying the following differential constraint

$$
\nabla_{\alpha}^{(i} \nabla_{\beta}^{j)} \Phi=0 .
$$

However, the multiplet is on-shell in the flat case in the sense that the constraint (3.1) implies $\square \Phi \equiv \partial^{a} \partial_{a} \Phi=0$ and there is no description in terms of an unconstrained superfield for such a multiplet. Despite this, it is still possible to work out a candidate for the trace superfield $A^{\alpha i j k}$ for a supergravity theory involving a compensating tensor multiplet. ${ }^{20} \mathrm{We}$ present this candidate below.

We first observe that we can construct an appropriate primary field $A^{\alpha i j k}$ as follows

$$
A^{\alpha i j k}=\frac{\mathrm{i}}{3} \Phi \nabla_{\beta}^{(i} \mathbb{H}^{\alpha \beta j k)}+\mathrm{i}\left(\nabla_{\beta}^{(i} \Phi\right) \mathbb{H}^{\alpha \beta j k)},
$$

\footnotetext{
${ }^{20}$ The fact that it exists is related to the fact that there is an invariant which is essentially a product of the tensor multiplet and a gauge three-form multiplet [41].
} 
where $\mathbb{H}^{\alpha \beta i j}=\mathbb{H}^{[\alpha \beta](i j)}$ is a primary superfield of dimension 3. Now we need to impose additional constraints on $\mathbb{H}^{\alpha \beta i j}$ in order for the trace superfield $A^{\alpha i j k}$ to both satisfy the consistency condition (2.5) and imply the existence of a conserved supersymmetry current. One can show that the consistency condition (2.5) is satisfied if we impose the constraint

$$
\nabla_{\alpha}^{(i} \mathbb{H}^{\beta \gamma j k)}=-\frac{2}{3} \delta_{\alpha}^{[\beta} \nabla_{\delta}^{(i} \mathbb{H}^{\gamma] \delta j k)} .
$$

One can check that eq. (3.3) is a primary constraint.

There exists another primary constraint that one can impose on $\mathbb{H}^{\alpha \beta i j}$ and it is

$$
\nabla_{\alpha}^{(i} \nabla_{\beta k} \mathbb{H}^{\alpha \beta j) k}+3 \mathrm{i} \nabla_{\alpha \beta} \mathbb{H}^{\alpha \beta i j}=0
$$

The constraints (3.3) and (3.4) are exactly the primary constraints that ensure $\mathbb{H}^{\alpha \beta i j}$ describes the lowest dimension component of a closed four-form [48].

One can check that in the flat case with the tensor multiplet set to unity, i.e. $\Phi=1$, and using the constraint (3.4), the descendent $A^{i j}$, defined by (2.7a), is

$$
A^{i j}=\frac{3}{16} D_{\alpha k} A^{\alpha i j k}=\frac{1}{4} \partial_{\alpha \beta} \mathbb{H}^{\alpha \beta i j} .
$$

Since $A^{i j}$ is a divergence we have a conserved $\mathrm{SU}(2)$ current, together with a conserved supersymmetry current and energy-momentum tensor according to the analysis of subsection 2.1. These are

$$
\begin{aligned}
j_{\alpha \beta}{ }^{i j} & =V_{\alpha \beta}{ }^{i j}-\mathrm{i} \mathbb{H}_{\alpha \beta}{ }^{i j}, & \partial^{\alpha \beta} j_{\alpha \beta}{ }^{i j} & =0, \\
S_{\alpha \beta, \gamma}{ }^{i} & =\Sigma_{\gamma, \alpha \beta}^{i}-\frac{2 \mathrm{i}}{3} D_{\gamma j} \mathbb{H}_{\alpha \beta}{ }^{i j}, & \partial^{\alpha \beta} S_{\alpha \beta, \gamma}^{i} & =0, \\
T_{\alpha \beta, \gamma \delta} & =\mathcal{T}_{\alpha \beta, \gamma \delta}-\frac{\mathrm{i}}{6} D_{\alpha i} D_{\beta j} \mathbb{H}_{\gamma \delta}{ }^{i j}-\frac{\mathrm{i}}{6} D_{\gamma i} D_{\delta j} \mathbb{H}_{\alpha \beta}{ }^{i j}, & \partial^{\alpha \beta} T_{\alpha \beta, \gamma \delta} & =0 .
\end{aligned}
$$

One should note that the supersymmetry current is not gamma-traceless and neither is the energy-momentum tensor traceless. To prove conservation of the energy-momentum tensor one uses

$$
\partial^{\alpha \beta} D_{\alpha i} D_{\beta j} \mathbb{H}_{\gamma \delta}{ }^{i j}=\partial^{\alpha \beta} D_{\gamma i} D_{\delta j} \mathbb{H}_{\alpha \beta}{ }^{i j},
$$

which follows from the differential constraints on $\mathbb{H}_{\alpha \beta}{ }^{i j}$, eqs. (3.3) and (3.4).

Remarkably, if we deform the constraint defining the tensor multiplet to

$$
\nabla_{\alpha}^{(i} \nabla_{\beta}^{j)} \Phi=\mathrm{i} \mathbb{H}_{\alpha \beta}{ }^{i j}
$$

the postulated superfield (3.2) still satisfies the consistency condition (2.5) but a conserved supersymmetry current is no longer guaranteed.

\subsection{An example: non-abelian gauge theory involving a compensating tensor multiplet}

For an illustrative example of a non-conformal supercurrent in curved superspace, we consider non-abelian gauge theory involving a compensating tensor multiplet. We refer the 
reader to appendix $\mathrm{C}$ for details on the description of the Yang-Mills multiplet in conformal superspace. The action for the theory is composed of two parts: (i) a higher-derivative nonabelian vector multiplet action; and (ii) the Yang-Mills action which involves the tensor multiplet and contains the term $\sigma \operatorname{Tr}\left(\boldsymbol{f}^{a b} \boldsymbol{f}_{a b}\right)$ at the component level [46]. Here $\sigma$ is the component projection of $\Phi$ and $\boldsymbol{f}_{a b}$ is the field strength of the non-abelian gauge field.

The higher-derivative non-abelian vector multiplet action is the non-abelian extension of the supersymmetric $F \square F$ action mentioned in subsection 2.2. It was described in Minkowski superspace in [44] and in conformal superspace in [41]. The supercurrent is

$$
J=\frac{3}{8} \operatorname{Tr}\left(X^{i j} X_{i j}+\frac{4 \mathrm{i}}{3} W^{\alpha i} \nabla_{\alpha \beta} W_{i}^{\beta}+\frac{2}{3} F_{\alpha}{ }^{\beta} F_{\beta}{ }^{\alpha}\right) .
$$

It is the unique dimension four primary superfield quadratic in the fields of the vector multiplet. The equation of motion for the vector multiplet is

$$
\mathcal{G}^{i j}=0,
$$

where we have introduced the superfield

$$
\begin{aligned}
\mathcal{G}^{i j}:= & \nabla^{a} \nabla_{a} X^{i j}-2 \mathrm{i}\left[W^{\alpha(i}, \nabla_{\alpha \beta} W^{\beta j)}\right]-\frac{3}{2}\left[X^{k(i}, X_{k}^{j)}\right]-2 Y_{\alpha}{ }^{\beta i j} F_{\beta}{ }^{\alpha} \\
& +\frac{5}{2} X^{\alpha(i} \overleftrightarrow{\nabla}_{\alpha \beta} W^{\beta j)},
\end{aligned}
$$

which is constructed to be primary of dimension 4 and to satisfy $\nabla_{\alpha}^{(i} \mathcal{G}^{j k)}=0 .{ }^{21}$ It can be checked that the supercurrent (3.9) is conserved,

$$
\nabla^{\alpha i j k} J=\mathrm{i} \operatorname{Tr}\left(W^{\alpha(i} \mathcal{G}^{j k)}\right)=0,
$$

as a consequence of the equation of motion (3.10).

We can now check the non-conformal conservation condition if we consider the higherderivative non-abelian vector multiplet action coupled to the Yang-Mills action. The YangMills action was described in conformal superspace in [41] by making use of a closed six-form with the lowest component given by the primary superfield

$$
A_{\alpha}^{i j k}=\varepsilon_{\alpha \beta \gamma \delta} V^{\beta(i} H^{\gamma \delta j k)}, \quad H^{\alpha \beta i j}:=\mathrm{i} \operatorname{Tr}\left(W^{\alpha(i} W^{\beta j)}\right),
$$

where $V^{\alpha i}$ is the constrained prepotential for the tensor multiplet [36, 50], which satisfies ${ }^{22}$

$$
\nabla_{\alpha}^{(i} V^{\beta j)}-\frac{1}{4} \delta_{\alpha}^{\beta} \nabla_{\gamma}^{(i} V^{\gamma j)}=0, \quad \Phi=\nabla_{\alpha i} V^{\alpha i}, \quad K^{A} \Phi=0, \quad \mathbb{D} \Phi=2 \Phi
$$

It should be noted that the primary superfield $H^{\alpha \beta i j}$ satisfies the same differential constraints as $\mathbb{H}^{\alpha \beta i j}$ does.

\footnotetext{
${ }^{21} \mathrm{JN}$ is grateful to Daniel Butter for checking this result using the computer algebra program Cadabra.

${ }^{22}$ Invariance under gauge transformations of the prepotential was shown in [41].
} 
For the combined action incorporating the higher-derivative non-abelian vector multiplet action and the Yang-Mills action, the equation of motion for the vector multiplet becomes

$$
\mathcal{G}^{i j}+\lambda\left(\Phi X^{i j}+\mathrm{i}\left(\nabla_{\alpha}^{(i} \Phi\right) W^{\alpha j)}\right)=0
$$

where $\lambda$ is a coupling constant. We also need to know the supercurrent $J$ for the combined theory. Interestingly, the supercurrent $J$ does not obtain a contribution from the Yang-Mills action. The point is that such a supercurrent would have to be linear in $\Phi$ and quadratic in the fields of the vector multiplet and no such scalar superfield of dimension 4 exists. Therefore, much like the $B F$ invariant, the Yang-Mills action can have no dependence on the supergravity prepotential $H$ and one can use the supercurrent of the higher-derivative Yang-Mills action for the combined system. Now using the supercurrent (3.9) and the equation of motion we find

$$
\nabla^{\alpha i j k} J=\frac{\mathrm{i}}{3} \Phi \nabla_{\beta}^{(i} \mathbb{H}^{\alpha \beta j k)}+\mathrm{i}\left(\nabla_{\beta}^{(i} \Phi\right) \mathbb{H}^{\alpha \beta j k)}, \quad \mathbb{H}^{\alpha \beta i j}=\lambda H^{\alpha \beta i j},
$$

which verifies the non-conformal supercurrent equation in the presence of a compensating tensor multiplet, cf. (3.2).

\subsection{The dilaton-Weyl multiplet}

We now discuss some subtleties about the non-conformal supercurrent just presented. As we have seen in previous sections, the supercurrent may be understood in terms of the variation of an action with respect to the conformal supergravity prepotential and possibly the prepotential of some supermultiplet that is to take on the role as a compensator. However, we obviously bump into a problem when we choose the compensator to be a tensor multiplet which has no prepotential formulation.

The tensor multiplet is quite special because its defining constraint (3.1) allows one to express the super-Weyl tensor in terms of the fields of the tensor multiplet,

$$
W_{a b c}=-\frac{1}{4} H_{a b c}-\frac{\mathrm{i}}{32}\left(\tilde{\gamma}_{a b c}\right)^{\gamma \delta} \nabla_{\gamma}^{k} \nabla_{\delta k} \Phi,
$$

where $H_{a b c}$ is the three-form field strength of the tensor multiplet. One should keep in mind that the combined system, tensor + Weyl-multiplet, is off-shell (with $40+40$ degrees of freedom) and upon replacing the covariant fields of the Weyl multiplet with those of the tensor multiplet leads to what is known as the dilaton-Weyl or type II Weyl multiplet [46]. One expects that the dilaton-Weyl multiplet should possess a prepotential formulation, albeit potentially taking a different form than that of the standard Weyl multiplet. We do not derive the details of such a formulation here but we wish to emphasise some important points below.

It is instructive to consider a superconformal action that may be described in standard conformal supergravity without a tensor multiplet, which possesses the supercurrent $J$ with the usual conservation condition (2.2). ${ }^{23}$ We can always replace the fields of the standard

\footnotetext{
${ }^{23}$ An example is provided by the linear (or $\mathcal{O}(2)$ ) multiplet action [45, 46] where the supercurrent is given (up to normalisation) by $J=G$.
} 
Weyl multiplet in the action with those of the dilaton-Weyl multiplet, which involves the tensor multiplet and thus gives a new action. However, this should only lead to a rewriting of the conservation condition on the supercurrent:

$$
\nabla^{\alpha i j k} J=\nabla^{\alpha i j k}\left(\Phi \frac{J}{\Phi}\right)=\frac{\mathrm{i}}{3} \Phi \nabla_{\beta}^{(i} \tilde{H}^{\alpha \beta j k)}+\mathrm{i}\left(\nabla_{\beta}^{(i} \Phi\right) \tilde{H}^{\alpha \beta j k)}=0,
$$

where

$$
\tilde{H}_{\alpha \beta}{ }^{i j}=\mathrm{i} \nabla_{\alpha}^{(i} \nabla_{\beta}^{j)}\left(\frac{J}{\Phi}\right)
$$

and $\tilde{H}_{\alpha \beta}{ }^{i j}$ satisfies the differential constraints (3.3) and (3.4). We see that for every such theory there always exists a superfield $\tilde{H}^{\alpha \beta i j}$ subject to the conservation condition (3.18).

The observation that the conservation of the supercurrent $J$ can be rewritten in terms of the superfield $\tilde{H}^{\alpha \beta i j}$ is important since the Yang-Mills action does not possess a supercurrent $J$ as discussed earlier. One can instead understand the superfield $H^{\alpha \beta i j}=\mathrm{i} \operatorname{Tr}\left(W^{\alpha(i} W^{\beta j)}\right)$ as the supercurrent in the dilaton-Weyl multiplet. This is consistent with the fact that the action is linear in the tensor multiplet and built out of covariant derivatives of $H^{\alpha \beta i j}$. Furthermore, the superfield $H^{\alpha \beta i j}$ corresponding to the Yang-Mills action satisfies the conservation condition (3.18) when the equation of motion for the Yang-Mills multiplet is enforced.

For the reasons mentioned above, one should think of the superfield $J^{\alpha \beta i j}$ satisfying the constraints (3.3) and (3.4) (with $\mathbb{H}^{\alpha \beta i j}$ replaced with $J^{\alpha \beta i j}$ ) and the on-shell conservation condition

$$
\frac{\mathrm{i}}{3} \Phi \nabla_{\beta}^{(i} J^{\alpha \beta j k)}+\mathrm{i}\left(\nabla_{\beta}^{(i} \Phi\right) J^{\alpha \beta j k)}=0
$$

as the supercurrent for a theory coupled to the dilaton-Weyl multiplet.

The dilaton-Weyl multiplet is expected to be described by an unconstrained prepotential $h^{\alpha \beta}{ }_{i j}$ such that its infinitesimal displacement generates the following variation of an action

$$
\delta S=\int \mathrm{d}^{6 \mid 8} z E \delta h^{\alpha \beta}{ }_{i j} J_{\alpha \beta}{ }^{i j} .
$$

The constraints (3.3) and (3.4) imposed on the supercurrent $J_{\alpha \beta}{ }^{i j}$ should be the conditions of the invariance of the action $S$ under certain gauge transformations of the gravitation superfield $h^{\alpha \beta}{ }_{i j}$. In fact these conditions follow from the gauge transformations

$$
\delta h_{\alpha \beta}^{i j}=\nabla_{\gamma k} \Lambda_{\alpha \beta}^{\gamma i j k}+\mathrm{i} \nabla_{[\alpha}^{(i} \nabla_{\beta] k} \Lambda^{j) k}-3 \nabla_{\alpha \beta} \Lambda^{i j},
$$

where the gauge parameters are primary and satisfy the conditions

$$
\Lambda_{\alpha \beta}^{\gamma i j k}=\Lambda_{[\alpha \beta]}^{\gamma(i j k)}, \quad \Lambda_{\alpha \beta}^{\beta i j k}=0, \quad \Lambda^{i j}=\Lambda^{(i j)} .
$$

The conservation condition (3.20) follows from the supergravity gauge transformations

$$
\delta h_{\alpha \beta}{ }^{i j}=\mathrm{i} \Phi \nabla_{[\alpha k} \Lambda_{\beta]}{ }^{i j k}-2 \mathrm{i}\left(\nabla_{[\alpha k} \Phi\right) \Lambda_{\beta]}{ }^{i j k}, \quad \Lambda_{\alpha}{ }^{i j k}=\Lambda_{\alpha}{ }^{(i j k)} .
$$

One can see that in the Minkowski superspace limit, the supercurrent $J^{\alpha \beta i j}$ satisfies the constraints

$$
D_{\alpha}^{(i} J^{\beta \gamma j k)}=0, \quad \partial^{\alpha \beta} J_{\alpha \beta}^{i j}=0
$$


Corresponding to the supercurrent put forward in $[4,51]$. One can also check that $J^{\alpha \beta i j}$ possesses $40+40$ degrees of freedom.

Suppose a matter action $S$ couples to a compensator, for instance the linear multiplet. Then the conservation equation (3.20) gets deformed to take the form

$$
\frac{\mathrm{i}}{3} \Phi \nabla_{\beta}^{(i} J^{\alpha \beta j k)}+\mathrm{i}\left(\nabla_{\beta}^{(i} \Phi\right) J^{\alpha \beta j k)}=A^{\alpha i j k}
$$

The consistency condition (2.5) follows from the above conservation condition keeping in mind the constraints (3.3) and (3.4) imposed on $J^{\alpha \beta i j}$, as well as the constraint (3.1) on $\Phi$. Using the results of subsection 2.1, we find that in the Minkowski superspace limit with $\Phi=1$

$$
\partial^{\alpha \beta} J_{\alpha \beta}^{i j}=-4 A^{i j}, \quad \partial^{\alpha \beta} \hat{\Sigma}_{\alpha \beta, \gamma}^{k}=4 \mathrm{i} \Lambda_{\gamma}^{k}, \quad \partial^{\alpha \beta} \hat{T}_{\alpha \beta, \gamma \delta}=4 \mathrm{i} \mathcal{A}_{\gamma \delta}
$$

where we have defined

$$
\hat{\Sigma}_{\alpha \beta, \gamma}^{i}:=-\frac{2 \mathrm{i}}{3} D_{\gamma j} J_{\alpha \beta}{ }^{i j}, \quad \hat{T}_{\alpha \beta, \gamma \delta}:=-\frac{\mathrm{i}}{6} D_{\alpha i} D_{\beta j} J_{\gamma \delta}{ }^{i j}-\frac{\mathrm{i}}{6} D_{\gamma i} D_{\delta j} J_{\alpha \beta}{ }^{i j} .
$$

This tells us that we still require $\Lambda_{\gamma}^{k}$ and $\mathcal{A}_{\gamma \delta}$ to be divergences in order for a conserved supersymmetry current and energy-momentum tensor to exist. However, now the supersymmetry current contains a gamma-trace component and the energy-momentum tensor contains a trace in addition to any contribution from the trace superfield $A^{\alpha i j k}$, which can be chosen to be any of the trace superfields derived in section 2 .

\section{The $\mathcal{N}=(2,0)$ non-conformal supercurrent}

In this section, we discuss the $\mathcal{N}=(2,0)$ superconformal current and put forward an $\mathcal{N}=(2,0)$ extension of the $\mathcal{N}=(1,0)$ non-conformal supercurrent based on a compensating tensor multiplet.

We first review some basic notation and conventions in regards to $\mathcal{N}=(2,0)$ supersymmetry. A symplectic Majorana spinor $\Psi_{i}$, decomposed as in [41], has Weyl components that satisfy the reality conditions

$$
\overline{\psi^{\alpha i}}=\psi_{i}^{\alpha}, \quad \overline{\chi_{\alpha i}}=\chi_{\alpha}^{i},
$$

where $i=1, \ldots, 4$ are $\mathrm{USp}(4)$ indices corresponding to the $R$-symmetry group. The USp(4) indices are raised and lowered as

$$
\Psi^{i}=\Omega^{i j} \Psi_{j}, \quad \Psi_{i}=\Omega_{i j} \Psi^{j}, \quad \Omega_{i j} \Omega^{j k}=\delta_{i}^{k},
$$

where $\Omega^{i j}=\Omega^{[i j]}$ is a symplectic metric of $\operatorname{USp}(4) \cdot{ }^{24}$ It satisfies

$$
\begin{aligned}
\varepsilon_{i j k l} & =3 \Omega_{i[j} \Omega_{k l]} \Longrightarrow \Omega^{i j}=-\frac{1}{2} \varepsilon^{i j k l} \Omega_{k l}, \\
\Omega^{[i j} \Phi^{k l]} & =\frac{1}{2}\left(\Omega^{[i j} \Phi^{k] l}-\Omega^{l[k} \Phi^{i j]}\right)=0,
\end{aligned}
$$

\footnotetext{
${ }^{24} \mathcal{N}=(1,0)$ is recovered by restricting $i=1,2$ and setting $\Omega^{i j}=\varepsilon^{i j}$.
} 
where $\Phi^{i j}=\Phi^{[i j]}$ is an antisymmetric rank 2 isospinor such that $\Phi^{i j} \Omega_{i j}=0$. Note that every antisymmetric rank $2 \mathrm{USp}(4)$ tensor $U^{i j}$ admits the decomposition $U^{i j}=\Phi^{i j}+\Omega^{i j} U$. Finally, the chiral $\mathcal{N}=(2,0)$ supersymmetry algebra is

$$
\left\{D_{\alpha}^{i}, D_{\beta}^{j}\right\}=-2 \mathrm{i} \Omega^{i j} \partial_{\alpha \beta} \equiv-2 \mathrm{i} \Omega^{i j}\left(\gamma^{a}\right)_{\alpha \beta} \partial_{a} .
$$

The $\mathcal{N}=(2,0)$ conformal supercurrent is described by the USp $(4)$ tensor superfield $J^{i j, k l}=J^{[i j],[k l]}=J^{k l, i j}=-2 J^{k[i, j] l}$, which is completely traceless with respect to the sympletic metric $\Omega_{i j}$ of $\operatorname{USp}(4)$, and satisfies the superspace conservation condition [4]

$$
D_{\alpha}^{m} J^{i j, k l}-\Omega^{m[i} \Psi_{\alpha}^{j], k l}-\frac{1}{4} \Omega^{i j} \Psi_{\alpha}^{m, k l}-\Omega^{m[k} \Psi_{\alpha}^{l], i j}-\frac{1}{4} \Omega^{k l} \Psi_{\alpha}^{m, i j}=0,
$$

where $\Psi_{\alpha}^{i, j k}=\Psi_{\alpha}^{i,[j k]}$ is completely traceless, $\Psi_{\alpha}^{i, j k} \Omega_{j k}=\Psi_{\alpha}^{i, j k} \Omega_{i j}=0$. The condition (4.5) is a constraint on the completely traceless part of $D_{\alpha}^{m} J^{i j, k l}$ and it fixes $\Psi_{\alpha}^{i, j k}=\frac{4}{7} D_{\alpha l} J^{l i, j k}$.

We can now insert a superfield $A_{\alpha}^{m, i j, k l}$ in the conservation equation (4.5) as follows: ${ }^{25}$

$$
D_{\alpha}^{m} J^{i j, k l}-\Omega^{m[i} \Psi_{\alpha}^{j], k l}-\frac{1}{4} \Omega^{i j} \Psi_{\alpha}^{m, k l}-\Omega^{m[k} \Psi_{\alpha}^{l], i j}-\frac{1}{4} \Omega^{k l} \Psi_{\alpha}^{m, i j}=A_{\alpha}^{m, i j, k l},
$$

where we require the trace superfield to satisfy the symmetry properties

$$
A_{\alpha}^{m, i j, k l}=A_{\alpha}^{m,[i j],[k l]}=A_{\alpha}^{m, k l, i j}, \quad A_{\alpha}^{m, i j, k l} \Omega_{i j}=A_{\alpha}^{m,[i j, k l]}=A_{\alpha}^{[m, i j], k l}=0,
$$

and the integrability condition ${ }^{26}$

$$
D_{\alpha}^{m} A_{\beta}^{n, i j, k l}+D_{\beta}^{n} A_{\alpha}^{m, i j, k l}-(\text { traces })=0,
$$

where (traces) represents all terms proportional to the metric $\Omega^{i j}$ consistent with the symmetry properties of $A_{\alpha}^{m, i j, k l}$.

We now put forward a candidate for the superfield $A_{\alpha}^{m, i j, k l}$ that is analogous to the non-conformal supercurrent based on a compensating $\mathcal{N}=(1,0)$ tensor multiplet. As a compensator, we will choose an $\mathcal{N}=(2,0)$ tensor multiplet HST83, which is described by an antisymmetric and $\Omega$-traceless superfield $\Phi^{i j}$,

$$
\Phi^{(i j)}=\Phi^{i j} \Omega_{i j}=0,
$$

satisfying the following differential constraint

$$
D_{\alpha}^{i} \Phi^{j k}-\Omega^{i[j} \lambda_{\alpha}^{k]}-\frac{1}{4} \Omega^{j k} \lambda_{\alpha}^{i}=0 .
$$

The constraint (4.10) eliminates the completely traceless part of $D_{\alpha}^{i} \Phi^{j k}$ and determines $\lambda_{\alpha}^{i}=\frac{4}{5} D_{\alpha j} \Phi^{j i}$.

Inspired by the $\mathcal{N}=(1,0)$ case, we write down the following candidate for the trace superfield

$$
A_{\alpha}^{m, i j, k l}=\Phi^{i j} H_{\alpha}^{m, k l}+\Phi^{k l} H_{\alpha}^{m, i j}-(\text { traces }),
$$

\footnotetext{
${ }^{25}$ This was also considered in [21] but with only a partial solution.

${ }^{26}$ This condition follows from requiring closure of supersymmetry on $J^{i j, k l}$.
} 
where $H_{\alpha}^{i, j k}=H_{\alpha}^{i,[j k]}$ is completely traceless and satisfies the constraint

$$
D_{\alpha}^{i} H_{\beta}^{j, k l}+D_{\beta}^{j} H_{\alpha}^{i, k l}-(\text { traces })=0 .
$$

The above constraint ensures that $A_{\alpha}^{m, i j, k l}$ satisfies its integrability condition (4.8). It is important to mention that the superfield $H_{\alpha}^{i, j k}$ is still very long and should be constrained to ensure the existence of a conserved supersymmetry current. In analogy with the $\mathcal{N}=$ $(1,0)$ case, we can constrain $H_{\alpha}^{i, j k}$ to be the lowest component of a closed four-form. This ensures that there exists an $\mathcal{N}=(1,0)$ component field which is the lowest component of a four-form in the $\mathcal{N}=(1,0)$ case. The postulated trace superfield (4.11) is expected to be the $\mathcal{N}=(2,0)$ extension of the one in the $\mathcal{N}=(1,0)$ case. The additional constraints on $H_{\alpha}^{i, j k}$ and the closed superform are described in appendix D.

\section{Discussion}

In this paper, we have presented various non-conformal supercurrents by finding solutions to the deformed conservation equation (2.3). Remarkably, we have managed to uncover an infinite number of solutions that are based on $\mathcal{O}(n)$ multiplets. The $n=2$ case corresponds to choosing the well-known linear multiplet as a compensator. For $n>2$ the possible compensators have not been extensively considered in detail before. Nevertheless, their usefulness was demonstrated in the description of the relaxed hypermultiplet given in this paper and such compensators can be used in the description of higher derivative actions (see the discussion section of [49]). In this light, it would be interesting if our results could be used to derive the equations of motion for higher derivative actions. Furthermore, the results in this paper should have analogues in lower dimensions and it would be interesting to work out their details in future work.

We explored the curious case of using the tensor multiplet as a compensator in section 3. Since coupling the Weyl multiplet to a tensor multiplet leads to a variant version of the Weyl multiplet, called the dilaton-Weyl multiplet, the supercurrent for the combined system needed to be modified. As an application of this supercurrent, we give the superspace equations of motion for minimal Poincaré supergravity [52] below.

Minimal Poincaré supergravity is derived from the action for the linear (or $\mathcal{O}(2)$ ) multiplet in conformal supergravity [46], which in superspace can be described by the full superspace integral

$$
S_{\mathrm{L}}:=\int \mathrm{d}^{6} x \mathrm{~d}^{8} \theta E \rho_{\alpha i} \mathbb{W}^{\alpha i}
$$

Here $\rho_{\alpha i}$ is the prepotential for the linear multiplet and $\mathbb{W}^{\alpha i}$ corresponds to an off-shell vector multiplet built out of the fields of the linear multiplet as follows [53]

$$
\begin{aligned}
\mathbb{W}^{\alpha i}= & \frac{1}{G} \nabla^{\alpha \beta} \Upsilon_{\beta}^{i}+\frac{4}{G}\left(W^{\alpha \beta} \Upsilon_{\beta}^{i}+10 \mathrm{i} X_{j}^{\alpha} G^{i j}\right)-\frac{1}{2 G^{3}} G_{j k}\left(\nabla^{\alpha \beta} G^{i j}\right) \Upsilon_{\beta}^{k} \\
& +\frac{1}{2 G^{3}} G^{i j} F^{\alpha \beta} \Upsilon_{\beta j}+\frac{\mathrm{i}}{16 G^{5}} \Upsilon_{\beta j} \Upsilon_{\gamma k} \Upsilon_{\delta l} G^{i j} G^{k l}
\end{aligned}
$$


where we have defined $\Upsilon_{\alpha}^{i}:=\frac{2}{3} \nabla_{\alpha j} G^{i j}$ and $F_{\alpha \beta}:=\frac{i}{4} \nabla_{[\alpha}^{k} \Upsilon_{\beta] k}$. The equations of motion in the standard Weyl multiplet read ${ }^{27}$

$$
\mathbb{W}^{\alpha i}=0, \quad J \propto G=0 .
$$

These equations of motion are obviously incompatible with the requirement that the linear multiplet is a conformal compensator since $G$ needs to be set to a non-vanishing constant. To remedy this, we should instead replace the Weyl multiplet with the dilaton-Weyl multiplet. Upon doing so, the equations of motion become

$$
\mathbb{W}^{\alpha i}=0, \quad \mathbb{H}_{\alpha \beta}{ }^{i j} \propto \nabla_{\alpha}^{(i} \nabla_{\beta}^{j)}\left(\frac{G}{\Phi}\right)=0,
$$

which are now consistent. The superspace equations of motion for gauged minimal supergravity [54] can be written down by considering a linear combination of the linear multiplet, the $B F$ and the Yang-Mills multiplet actions, and using the results in this paper. They are

$$
\begin{aligned}
\mathbb{W}^{\alpha i} & =-2 g W^{\alpha i}, \\
\left.\Phi X^{i j}+\mathrm{i}\left(\nabla_{\alpha}^{(i} \Phi\right) W^{\alpha j}\right) & =-g G^{i j}, \\
\frac{1}{4} \varepsilon^{\alpha \beta \gamma \delta} \nabla_{\gamma}^{(i} \nabla_{\delta}^{j)}\left(\frac{G}{\Phi}\right) & =W^{\alpha(i} W^{\beta j)},
\end{aligned}
$$

where $W^{\alpha i}$ describes an abelian vector multiplet, and $g$ is a coupling constant.

It is worth discussing the results in this paper in the context of Weyl anomalies. When one lifts a classical conformal field theory to curved space the resulting theory remains independent of any compensating scalar field. However, the conformal symmetry is anomalous at the quantum level. In the Weyl invariant formulation for gravity, the presence of conformal (or Weyl) anomalies is equivalent to the fact that the effective action acquires dependence on some compensator. The situation with supersymmetric field theories is analogous. Given a superconformal field theory, its action is independent of any compensator. The presence of superconformal (or super-Weyl) anomalies is equivalent to the fact that the effective action acquires dependence on a special compensator. In the case of 4D $\mathcal{N}=1$ superconformal theories, it was argued in [9] that the chiral scalar compensator of old minimal supergravity couples to the super-Weyl anomalies. The $4 \mathrm{D} \mathcal{N}=1$ super-Weyl anomalies were studied in $[55,56]$. For $4 \mathrm{D} \mathcal{N}=2$ superconformal theories, the super-Weyl anomalies are associated with the vector multiplet compensator [57].

It is natural to ask if any of the non-conformal supercurrents correspond to those associated with the super-Weyl anomalies in six dimensions. Here it is important to realise that unlike in four dimensions, the super-Weyl anomalies in six dimensions should be

\footnotetext{
${ }^{27}$ The first equation can, in principle, be derived by varying the action with respect to $\rho_{\alpha i}$; this is tedious because of the explicit $\rho$-dependence of $\mathbb{W}^{\alpha i}$. Alternatively, one can construct the most general primary field with the same index structure and weight $(3 / 2)$ as $\mathbb{W}^{\alpha i}$. The equation of motion for the supergravity multiplet is, $\frac{\delta S}{\delta H} \equiv J=0$. The supercurrent $J$ is a covariant expression built from the linear multiplet. Dimensional arguments fix it to be proportional to $G$.
} 
accompanied with Lorentz anomalies. However, each of the non-conformal supercurrents in this paper describe a conserved energy-momentum tensor with no Lorentz anomaly. This includes the non-conformal supercurrent corresponding to the conservation equation (1.9) and given in [21]. ${ }^{28}$ The absence of a Lorentz anomaly is evident from the fact that we assumed the supergravity actions were invariant under supergravity gauge transformations. Therefore, we need to change our set-up and this will be discussed elsewhere.

\section{Acknowledgments}

JN is grateful to Daniel Butter for useful discussions. SMK and JN thank the Galileo Galilei Institute for Theoretical Physics for hospitality and the INFN for partial support in September 2016. SMK acknowledges the Albert Einstein Institute for warm hospitality during part of this work. The work of SMK is supported in part by the Australian Research Council, project No. DP160103633. JN is supported by a Humboldt research fellowship of the Alexander von Humboldt Foundation. JN and ST acknowledge support from GIF the German-Israeli Foundation for Scientific Research and Development.

\section{A The geometry of $\mathcal{N}=(1,0)$ conformal superspace in six dimensions}

Here we collect the essential details of the superspace geometry of [41]. We refer the reader to appendix A of [41] for our notation and conventions.

We begin with a curved six-dimensional $\mathcal{N}=(1,0)$ superspace $\mathcal{M}^{6 \mid 8}$ parametrized by local bosonic $\left(x^{m}\right)$ and fermionic coordinates $\left(\theta_{i}\right)$ :

$$
z^{M}=\left(x^{m}, \theta_{i}^{\mu}\right)
$$

where $m=0,1, \cdots, 5, \mu=1, \cdots, 4$ and $i=1,2$. The structure group is chosen to be the full $6 \mathrm{D} \mathcal{N}=(1,0)$ superconformal group and the covariant derivatives are postulated to have the form

$$
\nabla_{A}=E_{A}-\frac{1}{2} \Omega_{A}^{a b} M_{a b}-\Phi_{A}^{k l} J_{k l}-B_{A} \mathbb{D}-\mathfrak{F}_{A B} K^{B}
$$

Here $E_{A}=E_{A}{ }^{M} \partial_{M}$ is the inverse vielbein, $M_{a b}$ are the Lorentz generators, $J^{i j}$ are generators of the $\mathrm{SU}(2)$ group, $\mathbb{D}$ is the dilatation generator and $K^{A}=\left(K^{a}, S_{i}^{\alpha}\right)$ are the special superconformal generators. We associate the Lorentz $\Omega_{A}{ }^{a b}, \mathrm{SU}(2) \Phi_{A}{ }^{k l}$, dilatation $B_{A}$ and special conformal $\mathfrak{F}_{A B}$ connections with their respective generators.

The Lorentz generators obey

$$
\begin{aligned}
{\left[M_{a b}, M_{c d}\right] } & =2 \eta_{c[a} M_{b] d}-2 \eta_{d[a} M_{b] c}, \\
{\left[M_{a b}, \nabla_{c}\right] } & =2 \eta_{c[a} \nabla_{b]}, \\
{\left[M_{\alpha}{ }^{\beta}, \nabla_{\gamma}^{k}\right] } & =-\delta_{\gamma}^{\beta} \nabla_{\alpha}^{k}+\frac{1}{4} \delta_{\alpha}^{\beta} \nabla_{\gamma}^{k} .
\end{aligned}
$$

\footnotetext{
${ }^{28}$ In this sense it is very much like its counterpart described by (1.9), which does not couple to the $4 \mathrm{D}$ $\mathcal{N}=2$ super-Weyl anomalies [57].
} 
The $\mathrm{SU}(2)$ and dilatation generators obey

$$
\begin{aligned}
{\left[J^{i j}, J^{k l}\right] } & =\varepsilon^{k(i} J^{j) l}+\varepsilon^{l(i} J^{j) k}, & {\left[J^{i j}, \nabla_{\alpha}^{k}\right] } & =\varepsilon^{k(i} \nabla_{\alpha}^{j)}, \\
{\left[\mathbb{D}, \nabla_{a}\right] } & =\nabla_{a}, & {\left[\mathbb{D}, \nabla_{\alpha}^{i}\right] } & =\frac{1}{2} \nabla_{\alpha}^{i} .
\end{aligned}
$$

The Lorentz and $\mathrm{SU}(2)$ generators act on the special conformal generators $K^{A}$ as

$$
\begin{array}{rlrl}
{\left[M_{a b}, K_{c}\right]} & =2 \eta_{c[a} K_{b]}, & {\left[M_{\alpha}^{\beta}, S_{k}^{\gamma}\right]=\delta_{\alpha}^{\gamma} S_{k}^{\beta}-\frac{1}{4} \delta_{\alpha}^{\beta} S_{k}^{\gamma},} \\
{\left[J^{i j}, S_{k}^{\gamma}\right]} & =\delta_{k}^{(i} S^{\gamma j)}, & &
\end{array}
$$

while the dilatation generator acts on $K_{A}$ as

$$
\left[\mathbb{D}, K_{a}\right]=-K_{a}, \quad\left[\mathbb{D}, S_{i}^{\alpha}\right]=-\frac{1}{2} S_{i}^{\alpha} .
$$

Among themselves, the generators $K_{A}$ obey the algebra

$$
\left\{S_{i}^{\alpha}, S_{j}^{\beta}\right\}=-2 \mathrm{i} \varepsilon_{i j}\left(\tilde{\gamma}^{c}\right)^{\alpha \beta} K_{c} .
$$

Finally, the algebra of $K_{A}$ with $\nabla_{A}$ is given by

$$
\begin{aligned}
{\left[K_{a}, \nabla_{b}\right] } & =2 \eta_{a b} \mathbb{D}+2 M_{a b}, & {\left[K_{a}, \nabla_{\alpha}^{i}\right] } & =-\mathrm{i}\left(\gamma_{a}\right)_{\alpha \beta} S^{\beta i}, \\
{\left[S_{i}^{\alpha}, \nabla_{a}\right] } & =-\mathrm{i}\left(\tilde{\gamma}_{a}\right)^{\alpha \beta} \nabla_{\beta i}, & \left\{S_{i}^{\alpha}, \nabla_{\beta}^{j}\right\} & =2 \delta_{\beta}^{\alpha} \delta_{i}^{j} \mathbb{D}-4 \delta_{i}^{j} M_{\beta}{ }^{\alpha}+8 \delta_{\beta}^{\alpha} J_{i}{ }^{j}
\end{aligned}
$$

The covariant derivatives obey (anti-)commutation relations of the form

$$
\begin{aligned}
{\left[\nabla_{A}, \nabla_{B}\right\}=} & -T_{A B}{ }^{C} \nabla_{C}-\frac{1}{2} R(M)_{A B}{ }^{c d} M_{c d}-R(J)_{A B}{ }^{k l} J_{k l} \\
& -R(\mathbb{D})_{A B} \mathbb{D}-R(S)_{A B}{ }^{k} S_{k}^{\gamma}-R(K)_{A B c} K^{c}
\end{aligned}
$$

where $T_{A B}^{C}$ is the torsion, and $R(M)_{A B}{ }^{c d}, R(J)_{A B}{ }^{k l}, R(\mathbb{D})_{A B}, R(S)_{A B \gamma}^{K}$ and $R(K)_{A B c}$ are the curvatures corresponding to the Lorentz, $\mathrm{SU}(2)$, dilatation, $S$-supersymmetry and special conformal boosts, respectively.

The full gauge group of conformal supergravity, $\mathcal{G}$, is generated by covariant general coordinate transformations, $\delta_{\text {cgct }}$, associated with a parameter $\xi^{A}$ and standard superconformal transformations, $\delta_{\mathcal{H}}$, associated with a parameter $\Lambda^{\underline{a}}$. The latter include the dilatation, Lorentz, SU(2), and special conformal (bosonic and fermionic) transformations. The covariant derivatives transform as

$$
\delta_{\mathcal{G}} \nabla_{A}=\left[\mathcal{K}, \nabla_{A}\right],
$$

where $\mathcal{K}$ denotes the first-order differential operator

$$
\mathcal{K}=\xi^{C} \nabla_{C}+\frac{1}{2} \Lambda^{a b} M_{a b}+\Lambda^{i j} J_{i j}+\Lambda \mathbb{D}+\Lambda_{A} K^{A} .
$$

Covariant (or tensor) superfields transform as

$$
\delta_{\mathcal{G}} T=\mathcal{K} T
$$


To describe conformal supergravity, the covariant derivative algebra (A.4) must be constrained as [41]

$$
\begin{aligned}
\left\{\nabla_{\alpha}^{i}, \nabla_{\beta}^{j}\right\}= & -2 \mathrm{i} \varepsilon^{i j}\left(\gamma^{a}\right)_{\alpha \beta} \nabla_{a} \\
{\left[\nabla_{a}, \nabla_{\alpha}^{i}\right]=} & \left(\gamma_{a}\right)_{\alpha \beta}\left(W^{\beta \gamma} \nabla_{\gamma}^{i}+4 \mathrm{i} X_{\delta}^{i \beta \gamma} M_{\gamma}{ }^{\delta}-\frac{\mathrm{i}}{2} X^{\gamma i} M_{\gamma}{ }^{\beta}-5 \mathrm{i} X_{j}^{\beta} J^{i j}+\frac{5 \mathrm{i}}{4} X^{\beta i} \mathbb{D}\right. \\
& +\frac{\mathrm{i}}{4} Y_{\gamma}^{\beta i j} S_{j}^{\gamma}+\frac{\mathrm{i}}{4} \nabla_{\gamma \delta} W^{\delta \beta} S^{\gamma i}-\frac{5 \mathrm{i}}{16} Y S^{\beta i} \\
& \left.+\frac{\mathrm{i}}{3}\left(\gamma^{b c}\right)_{\delta}^{\gamma}\left(\nabla_{b} X_{\gamma}^{i \delta \beta}-\frac{3}{4} \delta_{\gamma}^{\beta} \nabla_{b} X^{\delta i}\right) K_{c}\right)
\end{aligned}
$$

where $W^{\alpha \beta}$ is the super-Weyl tensor, which satisfies

$$
W^{\alpha \beta}=W^{\beta \alpha}, \quad S_{k}^{\gamma} W^{\alpha \beta}=0, \quad \mathbb{D} W^{\alpha \beta}=W^{\alpha \beta},
$$

and the Bianchi identities

$$
\begin{aligned}
\nabla_{\alpha}^{(i} \nabla_{\beta}^{j)} W^{\gamma \delta} & =-\delta_{[\alpha}^{(\gamma} \nabla_{\beta]}^{(i} \nabla_{\rho}^{j)} W^{\delta) \rho}, \\
\nabla_{\alpha}^{k} \nabla_{\gamma k} W^{\beta \gamma}-\frac{1}{4} \delta_{\alpha}^{\beta} \nabla_{\gamma}^{k} \nabla_{\delta k} W^{\gamma \delta} & =8 \mathrm{i} \nabla_{\alpha \gamma} W^{\gamma \beta} .
\end{aligned}
$$

Here the descendents of $W^{\alpha \beta}$ are defined as

$$
\begin{aligned}
X_{\gamma}^{k \alpha \beta} & :=-\frac{\mathrm{i}}{4} \nabla_{\gamma}^{k} W^{\alpha \beta}-\delta_{\gamma}^{(\alpha} X^{\beta) k}, \quad X^{\alpha i}:=-\frac{\mathrm{i}}{10} \nabla_{\beta}^{i} W^{\alpha \beta}, \\
Y_{\alpha}^{\beta i j} & :=-\frac{5}{2}\left(\nabla_{\alpha}^{(i} X^{\beta j)}-\frac{1}{4} \delta_{\alpha}^{\beta} \nabla_{\gamma}^{(i} X^{\gamma j)}\right)=-\frac{5}{2} \nabla_{\alpha}^{(i} X^{\beta j)} \\
Y & :=\frac{1}{4} \nabla_{\gamma}^{k} X_{k}^{\gamma}, \\
Y_{\alpha \beta}^{\gamma \delta} & :=\nabla_{(\alpha}^{k} X_{\beta) k}^{\gamma \delta}-\frac{1}{6} \delta_{\beta}^{(\gamma} \nabla_{\rho}^{k} X_{\alpha k}^{\delta) \rho}-\frac{1}{6} \delta_{\alpha}^{(\gamma} \nabla_{\rho}^{k} X_{\beta k}{ }^{\delta) \rho} .
\end{aligned}
$$

Note that $X_{\gamma}^{k \alpha \beta}$ is traceless, $Y_{\alpha}^{\beta i j}$ is symmetric in its $\mathrm{SU}(2)$ indices and traceless in its spinor indices, and $Y_{\alpha \beta}{ }^{\gamma \delta}$ is separately symmetric in its upper and lower spinor indices and traceless.

Upon taking a spinor covariant derivative of the descendent fields one finds

$$
\begin{aligned}
\nabla_{\alpha}^{i} X^{\beta j}= & -\frac{2}{5} Y_{\alpha}^{\beta i j}-\frac{2}{5} \varepsilon^{i j} \nabla_{\alpha \gamma} W^{\gamma \beta}-\frac{1}{2} \varepsilon^{i j} \delta_{\alpha}^{\beta} Y \\
\nabla_{\alpha}^{i} X_{\beta}^{j \gamma \delta}= & \frac{1}{2} \delta_{\alpha}^{(\gamma} Y_{\beta}^{\delta) i j}-\frac{1}{10} \delta_{\beta}^{(\gamma} Y_{\alpha}^{\delta) i j}-\frac{1}{2} \varepsilon^{i j} Y_{\alpha \beta} \gamma \delta-\frac{1}{4} \varepsilon^{i j} \nabla_{\alpha \beta} W^{\gamma \delta} \\
& +\frac{3}{20} \varepsilon^{i j} \delta_{\beta}^{(\gamma} \nabla_{\alpha \rho} W^{\delta) \rho}-\frac{1}{4} \varepsilon^{i j} \delta_{\alpha}^{(\gamma} \nabla_{\beta \rho} W^{\delta) \rho}, \\
\nabla_{\alpha}^{i} Y= & -2 \mathrm{i} \nabla_{\alpha \beta} X^{\beta i} \\
\nabla_{\gamma}^{k} Y_{\alpha}^{\beta i j}= & \frac{2}{3} \varepsilon^{k(i}\left(-8 \mathrm{i} \nabla_{\gamma \delta} X_{\alpha}^{j) \delta \beta}-4 \mathrm{i} \nabla_{\alpha \delta} X_{\gamma}^{j) \delta \beta}+3 \mathrm{i} \nabla_{\gamma \alpha} X^{\beta j)}\right. \\
& \left.+3 \mathrm{i} \delta_{\gamma}^{\beta} \nabla_{\alpha \delta} X^{\delta j)}-\frac{3 \mathrm{i}}{2} \delta_{\alpha}^{\beta} \nabla_{\gamma \delta} X^{\delta j)}\right), \\
\nabla_{\epsilon}^{i} Y_{\alpha \beta}{ }^{\gamma \delta}= & \left.-4 \mathrm{i} \nabla_{\epsilon(\alpha} X_{\beta)}^{l} \gamma \delta+\frac{4 \mathrm{i}}{3} \delta_{(\alpha}^{(\gamma} \nabla_{\beta) \rho} X_{\epsilon}^{l \delta) \rho}+\frac{8 \mathrm{i}}{3} \delta_{(\alpha}^{(\gamma} \nabla_{|\epsilon \rho|} X_{\beta)}^{l} \delta\right) \rho \\
& \left.+8 \mathrm{i} \delta_{\epsilon}^{(\gamma} \nabla_{\rho(\alpha} X_{\beta)}^{l} \delta\right) \rho
\end{aligned}
$$


The descendant superfields transform under $S$-supersymmetry as follows:

$$
\begin{aligned}
& S_{i}^{\alpha} X_{\beta}^{j \gamma \delta}=-\mathrm{i} \delta_{i}^{j} \delta_{\beta}^{\alpha} W^{\gamma \delta}+\frac{2 \mathrm{i}}{5} \delta_{i}^{j} \delta_{\beta}^{(\gamma} W^{\delta) \alpha}, \quad S_{i}^{\alpha} X^{\beta j}=\frac{8 \mathrm{i}}{5} \delta_{i}^{j} W^{\alpha \beta}, \\
& S_{k}^{\gamma} Y_{\alpha}^{\beta i j}=\delta_{k}^{(i}\left(-16 X_{\alpha}^{j) \gamma \beta}+2 \delta_{\alpha}^{\beta} X^{\gamma j)}-8 \delta_{\alpha}^{\gamma} X^{\beta j)}\right), \\
& S_{j}^{\rho} Y_{\alpha \beta}{ }^{\gamma \delta}=24\left(\delta_{(\alpha}^{\rho} X_{\beta) j}{ }^{\gamma \delta}-\frac{1}{3} \delta_{(\alpha}^{(\gamma} X_{\beta) j}{ }^{\delta) \rho}\right), \quad S_{i}^{\alpha} Y=-4 X_{i}^{\alpha} .
\end{aligned}
$$

\section{B The prepotential for the $\mathcal{O}(2)$ multiplet}

The prepotential formulation for the $\mathcal{O}(2)$ multiplet was first given in Minkowski superspace in [45] in terms of a spinor superfield $\rho_{\alpha}^{i}$. In this appendix we extend the construction in [45] to supergravity by making use of projective superspace techniques. The projective superspace approach is a method to construct general off-shell supersymmetric theories with eight supercharges. Originally it was introduced in four and three dimensions $[33,35,58,59]$ and then extended to five [60] and six $[45,61,62]$ dimensions. The projective-superspace approach to conformal supergravity was first developed in five dimensions [39, 63] and then extended to four [40, 64, 65] and six dimensions [36].

In the projective superspace setting, the supermanifold $\mathcal{M}^{6 \mid 8}$ is augmented with an additional $\mathbb{C P}^{1}$ parametrized by an isotwistor coordinate $v^{i} \in \mathbb{C}^{2} \backslash\{0\}$. Matter fields are constructed in terms of covariant projective multiplets $\mathcal{Q}^{(n)}(z, v)$, which are holomorphic in the isotwistor $v^{i}$ and of definite homogeneity, $\mathcal{Q}^{(n)}(z, c v)=c^{n} \mathcal{Q}^{(n)}(z, v)$, on an open domain of $\mathbb{C}^{2} \backslash\{0\}$. Such superfields are intrinsically defined on $\mathbb{C P}^{1}$.

It is useful to introduce an additional fixed isotwistor $u_{i}$ which obeys $v^{i} u_{i} \neq 0$. Given a superfield $T^{i_{i} \cdots i_{n}}$ with symmetric SU(2) indices $T^{i_{i} \cdots i_{n}}=T^{\left(i_{i} \cdots i_{n}\right)}$ (and suppressed Lorentz indices) we define

$$
T^{(m)(m-n)}:=v_{i_{1}} \cdots v_{i_{m}} \frac{u_{i_{m+1}}}{(v, u)} \cdots \frac{u_{i_{n}}}{(v, u)} T^{i_{1} \cdots i_{n}}, \quad(v, u):=v^{k} u_{k} .
$$

We also introduce

$$
\nabla_{\alpha}^{(1)}:=v_{i} \nabla_{\alpha}^{i}, \quad \nabla_{\alpha}^{(-1)}=\frac{u_{i}}{(v, u)} \nabla_{\alpha}^{i}
$$

and the following derivative operations

$$
\partial^{(2)}=(v, u) v_{i} \frac{\partial}{\partial u_{i}}, \quad \partial^{(0)}=v^{i} \frac{\partial}{\partial v^{i}}-u_{i} \frac{\partial}{\partial u_{i}}, \quad \partial^{(-2)}=\frac{1}{(v, u)} u^{i} \frac{\partial}{\partial v^{i}} .
$$

Fields and operators of definite homogeneity in $v^{i}$ can be interpreted as possessing definite $\partial^{(0)}$ charge. Note that one can express the $\mathrm{SU}(2)$ generator in terms of the above derivative operators as follows

$$
J_{i j}=-v_{i} v_{j} \partial^{(-2)}+\frac{\left.v_{(i} u_{j}\right)}{(v, u)} \partial^{(0)}+\frac{1}{(v, u)^{2}} u_{i} u_{j} \partial^{(2)} .
$$

Let us now use the above isotwistor notation to write down a candidate for the prepotential description of the $\mathcal{O}(2)$ multiplet, $G^{(2)}=v_{i} v_{j} G^{i j}$, which satisfies the constraint

$$
\nabla_{\alpha}^{(1)} G^{(2)}=0 .
$$


Considering the generalisation of the result in [45] to supergravity, one is led to the following natural ansatz

$$
G^{(2)}=\nabla^{(4)}\left(\nabla^{\alpha(-3)} \rho_{\alpha}^{(1)}+a \mathrm{i} \nabla_{\alpha}^{(-1)} \nabla^{\alpha \beta} \rho_{\beta}^{(-1)}+b \mathrm{i} \nabla_{\alpha}^{(-1)}\left(W^{\alpha \beta} \rho_{\beta}^{(-1)}\right)\right),
$$

where $a$ and $b$ are some coefficients to be determined and $\rho_{\alpha}^{i}$ is the prepotential which we assume to be primary and of dimension $1 / 2$. Here we have introduced the covariant differential operators

$$
\nabla^{(4)}:=\frac{1}{4 !} \varepsilon^{\alpha \beta \gamma \delta} \nabla_{\alpha}^{(1)} \nabla_{\beta}^{(1)} \nabla_{\gamma}^{(1)} \nabla_{\delta}^{(1)}, \quad \nabla^{\alpha(3)}:=\frac{1}{3 !} \varepsilon^{\alpha \beta \gamma \delta} \nabla_{\alpha}^{(1)} \nabla_{\beta}^{(1)} \nabla_{\gamma}^{(1)} .
$$

For consistency we require independence of the isotwister $u^{i}$, which amounts to requiring

$$
\partial^{(2)} G^{(2)}=0 .
$$

Using the property $\nabla_{\alpha}^{(1)} \nabla^{(4)}=\nabla^{(4)} \nabla_{\alpha}^{(1)}=0$ and the above requirement fixes the coefficients $a$ and $b$ as

$$
G^{(2)}=\nabla^{(4)}\left(\nabla^{\alpha(-3)} \rho_{\alpha}^{(1)}-2 \mathrm{i} \nabla_{\alpha}^{(-1)} \nabla^{\alpha \beta} \rho_{\beta}^{(-1)}-8 \mathrm{i} \nabla_{\alpha}^{(-1)}\left(W^{\alpha \beta} \rho_{\beta}^{(-1)}\right)\right) .
$$

The prepotential is defined up to some gauge freedom. Specifically, $G^{(2)}$ is unchanged by the following shift in the prepotential

$$
\rho_{\alpha}^{i} \rightarrow \rho_{\alpha}^{i}=\rho_{\alpha}^{i}+\nabla_{\alpha}^{i} \tau+\nabla_{\beta j} \tau_{\alpha}^{\beta i j}, \quad \tau_{\alpha}^{\alpha i j}=0, \quad \tau_{\alpha}{ }^{\beta i j}=\tau_{\alpha}^{\beta(i j)},
$$

where $\tau$ and $\tau_{\alpha}{ }^{\beta i j}$ are dimensionless primary superfields. It is rather simple to show that the transformations associated with the scalar $\tau$ leave $G^{(2)}$ invariant since

$$
\left\{\nabla_{\alpha}^{(1)}, \nabla^{\alpha(-3)}\right\} \tau=2 \mathrm{i} \nabla_{\alpha}^{(-1)} \nabla^{\alpha \beta} \nabla_{\beta}^{(-1)} \tau+8 \mathrm{i} \nabla_{\alpha}^{(-1)}\left(W^{\alpha \beta} \nabla_{\beta}^{(-)} \tau\right) .
$$

To show that $G^{(2)}$ is also invariant with respect to the transformations associated with $\tau_{\alpha}^{\beta i j}$ one uses the identities

$$
\begin{aligned}
\nabla^{(4)} \nabla_{\alpha}^{(1)} & =0 \\
\nabla^{\alpha(-3)} \nabla_{\beta}^{(-1)} & =\delta_{\beta}^{\alpha} \nabla^{(-4)} \\
\left\{\nabla_{\alpha}^{(-1)} \nabla^{\alpha \beta}, \nabla_{\gamma}^{(1)}\right\} \tau_{\beta}^{\gamma(-2)}= & -4 \nabla_{\alpha}^{(-1)}\left(W^{\alpha \beta} \nabla_{\gamma}^{(1)} \tau_{\beta}^{\gamma(-2)}\right), \\
\left\{\nabla^{\alpha(-3)}, \nabla_{\beta}^{(1)}\right\} \tau_{\alpha}^{\beta(1)(-1)}= & -2 \mathrm{i} \nabla_{\alpha}^{(-1)} \nabla^{\alpha \beta} \nabla_{\gamma}^{(-1)} \tau_{\beta}^{\gamma(1)(-1)} \\
& -8 \mathrm{i} \nabla_{\alpha}^{(-1)}\left(W^{\alpha \beta} \nabla_{\gamma}^{(-1)} \tau_{\beta}^{\gamma(1)(-1)}\right) .
\end{aligned}
$$

We now have to check that $G^{(2)}$ is primary. To do so, we apply the following identities

$$
\begin{aligned}
{\left[S^{\alpha(-1)}, \nabla^{(4)}\right] } & =-12 \nabla^{\alpha(3)}+\nabla^{\beta(3)}\left(2 \delta_{\beta}^{\alpha} \mathbb{D}-4 M_{\beta}^{\alpha}-4 \delta_{\beta}^{\alpha} \partial^{(0)}\right), \\
\left\{S^{\alpha(-1)}, \nabla^{\beta(-3)}\right\} & =4 \varepsilon^{\alpha \beta \gamma \delta} \nabla_{\gamma}^{(-1)} \nabla_{\delta}^{(-1)} \partial^{(-2)} \\
\left\{S^{\alpha(-1)}, \nabla_{\gamma}^{(-1)} \nabla^{\gamma \beta}\right\} & =-8 \nabla^{\alpha \beta} \partial^{(-2)}-2 \mathrm{i} \varepsilon^{\alpha \beta \gamma \delta} \nabla_{\gamma}^{(-1)} \nabla_{\delta}^{(-1)}
\end{aligned}
$$


to show

$$
S^{\alpha(-1)} G^{(2)}=0 .
$$

Furthermore, since $G^{(2)}$ is independent of the isotwistor $u^{i}$ we must have

$$
S_{i}^{\alpha} G^{j k}=0
$$

and thus $G^{i j}$ is primary.

Finally, since $G^{(2)}$ is independent of the isotwistor $u^{i}, G^{i j}$ can be written without isotwistors as follows

$$
G^{i j}=\frac{1}{2} \nabla^{(i j k l} \nabla_{k l p}^{\alpha} \rho_{\alpha}^{p)}-\frac{6 \mathrm{i}}{5} \nabla^{i j k l}\left(\nabla_{\alpha k} \nabla^{\alpha \beta} \rho_{\beta l}+4 \nabla_{\alpha k}\left(W^{\alpha \beta} \rho_{\beta l}\right)\right) .
$$

\section{The Yang-Mills multiplet in conformal superspace}

In this appendix, we give the results needed for the description of the Yang-Mills multiplet in conformal superspace. It is based almost verbatim on appendix $\mathrm{C}$ of [41].

To describe a non-abelian vector multiplet, the covariant derivative $\nabla_{A}$ is replaced with a gauge covariant one,

$$
\nabla_{A}:=\nabla_{A}-\mathrm{i} V_{A}
$$

Here the gauge connection one-form $V_{A}$ takes its values in the Lie algebra of the (unitary) Yang-Mills gauge group, $G_{\mathrm{YM}}$, with its (Hermitian) generators commuting with all the generators of the superconformal algebra. The algebra of the gauge covariant derivatives is

$$
\begin{aligned}
{\left[\boldsymbol{\nabla}_{A}, \boldsymbol{\nabla}_{B}\right\}=} & -T_{A B}{ }^{C} \boldsymbol{\nabla}_{C}-\frac{1}{2} R(M)_{A B}{ }^{c d} M_{c d}-R(J)_{A B}{ }^{k l} J_{k l}-R(\mathbb{D})_{A B} \mathbb{D} \\
& -R(S)_{A B k}^{\gamma} S_{\gamma}^{k}-R(K)_{A B}{ }^{c} K_{c}-\mathrm{i} F_{A B},
\end{aligned}
$$

where the torsion and curvatures are those of conformal superspace and $F_{A B}$ is the gauge covariant field strength two-form. It satisfies the Bianchi identity

$$
\nabla_{[A} F_{B C\}}+T_{[A B}^{D} F_{|D| C\}}=0 .
$$

The Yang-Mills gauge transformation acts on the gauge covariant derivatives $\nabla_{A}$ and a matter superfield $U$ (transforming in some representation of the gauge group) as

$$
\nabla_{A} \rightarrow \mathrm{e}^{\mathrm{i} \tau} \nabla_{A} \mathrm{e}^{-\mathrm{i} \tau}, \quad U \rightarrow U^{\prime}=\mathrm{e}^{\mathrm{i} \tau} U, \quad \tau^{\dagger}=\tau,
$$

where the Hermitian gauge parameter $\tau(z)$ takes its values in the Lie algebra of $G_{\mathrm{YM}}$.

Some components of the superform field strength have to be constrained in order to describe an irreducible multiplet. In conformal superspace, the components of the field strength are constrained as

$$
\begin{aligned}
F_{\alpha \beta}^{i j} & =0, \\
F_{a \beta}^{j} & =\left(\gamma_{a}\right)_{\alpha \beta} W^{\beta i}, \\
F_{a b} & =-\frac{\mathrm{i}}{8}\left(\gamma_{a b}\right)_{\beta}{ }^{\alpha} \nabla_{\alpha}^{k} W_{k}^{\beta},
\end{aligned}
$$


where $W^{\alpha i}$ is a conformal primary of dimension $\frac{3}{2}, S_{k}^{\gamma} W^{\alpha i}=0$ and $\mathbb{D} W^{\alpha i}=\frac{3}{2} W^{\alpha i}$. The Bianchi identity (C.3) constrains $W^{\alpha i}$ to obey the differential constraints

$$
\boldsymbol{\nabla}_{\gamma}^{k} W_{k}^{\gamma}=0, \quad \nabla_{\alpha}^{(i} W^{\beta j)}=\frac{1}{4} \delta_{\alpha}^{\beta} \boldsymbol{\nabla}_{\gamma}^{(i} W^{\gamma j)} .
$$

It is useful to introduce the following descendant superfield:

$$
X^{i j}:=\frac{\mathrm{i}}{4} \nabla_{\gamma}^{(i} W^{\gamma j)} .
$$

The superfield $W^{\alpha i}$ and $X^{i j}$, together with

$$
F_{\alpha}{ }^{\beta}=-\frac{\mathrm{i}}{4}\left(\nabla_{\alpha}^{k} W_{k}^{\beta}-\frac{1}{4} \delta_{\alpha}^{\beta} \nabla_{\gamma}^{k} W_{k}^{\gamma}\right)=-\frac{\mathrm{i}}{4} \nabla_{\alpha}^{k} W_{k}^{\beta},
$$

satisfy the following identities:

$$
\begin{aligned}
\nabla_{\alpha}^{i} W^{\beta j} & =-\mathrm{i} \delta_{\alpha}^{\beta} X^{i j}-2 \mathrm{i} \varepsilon^{i j} F_{\alpha}^{\beta}, \\
\nabla_{\alpha}^{i} F_{\beta}^{\gamma} & =-\nabla_{\alpha \beta} W^{\gamma i}-\delta_{\alpha}^{\gamma} \nabla_{\beta \delta} W^{\delta i}+\frac{1}{2} \delta_{\beta}^{\gamma} \nabla_{\alpha \delta} W^{\delta i}, \\
\nabla_{\alpha}^{i} X^{j k} & =2 \varepsilon^{i(j} \nabla_{\alpha \beta} W^{\beta k)}, \\
S_{k}^{\gamma} F_{\alpha}{ }^{\beta} & =-4 \mathrm{i} \delta_{\alpha}^{\gamma} W_{k}^{\beta}+\mathrm{i} \delta_{\alpha}^{\beta} W_{k}^{\gamma}, \quad S_{k}^{\gamma} X^{i j}=-4 \mathrm{i} \delta_{k}^{(i} W^{\gamma j)} .
\end{aligned}
$$

\section{A superform description for the $\mathcal{N}=(2,0)$ tensor multiplet and its deformation}

Here we give the superform description for the $\mathcal{N}=(2,0)$ tensor multiplet and introduce a closed four-form by deforming the constraints defining the tensor multiplet. ${ }^{29}$

The tensor multiplet can be described by a two-form gauge potential in superspace [ 67 , 68]. The field strength three-form $H_{3}=\frac{1}{3 !} \mathrm{d} z^{C} \mathrm{~d} z^{B} \mathrm{~d} z^{A} H_{A B C}$ is given in terms of its twoform gauge potential $B_{2}=\frac{1}{2} \mathrm{~d} z^{B} \mathrm{~d} z^{A} B_{A B}$ by

$$
H_{3}=\mathrm{d} B_{2} \Longrightarrow H_{A B C}=3 D_{[A} B_{B C\}}+3 T_{[A B}^{D} B_{|D| C\}},
$$

where the only non-vanishing component of the torsion is

$$
T_{\alpha \beta}^{i j a}=2 \mathrm{i} \Omega^{i j}\left(\gamma^{a}\right)_{\alpha \beta}
$$

The existence of the gauge potential requires that the Bianchi identity

$$
\mathrm{d} H_{3}=0 \Longrightarrow D_{[A} H_{B C D\}}+\frac{3}{2} T_{[A B}{ }^{E} H_{|E| C D\}}=0
$$

be satisfied. To describe the tensor multiplet, one must impose the following constraints on the lowest components of the superform field strength:

$$
H_{\alpha \beta \gamma}^{i j k}=0, \quad H_{a \alpha \beta}^{i j}=2 \mathrm{i}\left(\gamma_{a}\right)_{\alpha \beta} \Phi^{i j}, \quad \Phi^{(i j)}=\Phi^{i j} \Omega_{i j}=0
$$

\footnotetext{
${ }^{29}$ The superform description of the $\mathcal{N}=(1,0)$ tensor multiplet was given in [66].
} 
The Bianchi identities for $H_{3}$ can then be solved giving the remaining components:

$$
\begin{aligned}
H_{a b \alpha}^{i} & =-\frac{1}{4}\left(\gamma_{a b}\right)_{\alpha}{ }^{\beta} \lambda_{\beta}^{i}, \\
H_{a b c} & =\frac{1}{8}\left(\tilde{\gamma}_{a b c}\right)^{\alpha \beta} H_{\alpha \beta}, \quad H_{[\alpha \beta]}=0,
\end{aligned}
$$

where $\Phi^{i j}$ is required to satisfy the differential constraint

$$
D_{\alpha}^{i} \Phi^{j k}-\Omega^{i[j} \lambda_{\alpha}^{k]}-\frac{1}{4} \Omega^{j k} \lambda_{\alpha}^{i}=0
$$

and its corollaries

$$
\begin{aligned}
D_{\alpha}^{i} \lambda_{\beta}^{j} & =2 \mathrm{i} \Omega^{i j} H_{\alpha \beta}+4 \mathrm{i} \partial_{\alpha \beta} \Phi^{i j}, \\
D_{\alpha}^{i} H_{\beta \gamma} & =\partial_{\alpha(\beta} \lambda_{\gamma)}^{i}, \quad \partial^{\alpha \beta} \lambda_{\beta}^{i}=0 .
\end{aligned}
$$

The constraint (D.5) is the defining constraint for the $\mathcal{N}=(2,0)$ tensor multiplet.

We now wish to describe a closed four-form $H_{4}=\frac{1}{4 !} \mathrm{d} z^{D} \mathrm{~d} z^{C} \mathrm{~d} z^{B} \mathrm{~d} z^{A} H_{A B C D}$, which satisfies the closure condition

$$
\mathrm{d} H_{4}=0 \Longrightarrow D_{[A} H_{B C D E\}}+2 T_{[A B}^{F} H_{|F| C D E\}}=0 .
$$

To do this we proceed by obstructing the constraint defining the tensor multiplet by a closed 4-form $\mathrm{H}_{4}$ as $^{30}$

$$
\mathrm{d} H_{3}=H_{4} \quad \Longrightarrow \quad 4 D_{[A} H_{B C D\}}+6 T_{[A B}^{E} H_{|E| C D\}}=H_{A B C D}
$$

such that the constraint on the tensor multiplet is deformed to

$$
D_{\alpha}^{i} \Phi^{j k}-\Omega^{i[j} \lambda_{\alpha}^{k]}-\frac{1}{4} \Omega^{j k} \lambda_{\alpha}^{i}=H_{\alpha}^{i, j k}
$$

where $H_{\alpha}^{i, j k}=H_{\alpha}^{i,[j k]}$ is completely traceless and the four-form is constructed completely in terms of $H_{\alpha}^{i, j k}$. The first non-vanishing component of $H_{4}$ is fixed by the condition (D.8) and the constraint (D.9) to be

$$
H_{a \alpha \beta \gamma}^{i j k}=-2 \mathrm{i}\left(\gamma_{a}\right)_{\alpha \beta} H_{\gamma}^{k, i j}-2 \mathrm{i}\left(\gamma_{a}\right)_{\beta \gamma} H_{\alpha}^{i, j k}-2 \mathrm{i}\left(\gamma_{a}\right)_{\gamma \alpha} H_{\beta}^{j, k i}
$$

The remaining components can be determined by finding the conditions that follow from the constraint (D.9) and using eq. (D.8). The consistency conditions on $H_{\alpha}^{i, j k}$ that follow from the constraint (D.9) will give the requirements for $H_{4}$ to be closed.

By taking successive spinor derivatives of the superfield $\Phi^{i j}$ one finds the following results at dimension 4 :

$$
\begin{gathered}
D_{\alpha}^{i} \lambda_{\beta}^{j}=H_{\alpha \beta}{ }^{i j}+2 \mathrm{i} \Omega^{i j} H_{\alpha \beta}+4 \mathrm{i} \partial_{\alpha \beta} \Phi^{i j}, \quad \Omega_{i j} H_{\alpha \beta}{ }^{i j}=0 \\
D_{\alpha}^{i} H_{\beta}^{j, k l}+D_{\beta}^{j} H_{\alpha}^{i, k l}=-\Omega^{i[k} H_{\beta \alpha}{ }^{|j| l]}-\Omega^{j[k} H_{\alpha \beta}{ }^{\mid i l]}-\frac{1}{4} \Omega^{k l} H_{\alpha \beta}{ }^{i j}-\frac{1}{4} \Omega^{k l} H_{\beta \alpha}{ }^{j i}
\end{gathered}
$$

\footnotetext{
${ }^{30} \mathrm{~A}$ similar procedure of obstructing the closure condition of a $p$-superform to obtain a closed $(p+1)$ superform was used in [48] to construct a chain of closed $\mathcal{N}=(1,0)$ superforms.
} 
while at dimension $9 / 2$ one finds

$$
\begin{aligned}
D_{\gamma}^{i} H_{\alpha \beta} & =\partial_{\gamma(\alpha} \lambda_{\beta)}^{i}-\frac{\mathrm{i}}{30} D_{\gamma k} H_{(\alpha \beta)}^{i k}+\frac{2 \mathrm{i}}{15} D_{(\alpha k} H_{|\gamma| \beta)}{ }^{i k}, \\
15 \mathrm{i} \partial_{[\alpha \beta} \lambda_{\gamma]}^{i} & =D_{\gamma k} H_{[\alpha \beta]}^{i k}-4 D_{[\alpha k} H_{|\gamma| \beta]}{ }^{i k} \\
30 \mathrm{i} \partial_{[\alpha \beta} \lambda_{\gamma]}^{i} & =D_{[\gamma k} H_{\alpha] \beta}{ }^{i k}-3 D_{[\gamma k} H_{\alpha] \beta}^{k i} \\
0 & =D_{\alpha}^{i} H_{\beta \gamma}{ }^{j k}+4 \mathrm{i} \partial_{\beta \gamma} H_{\alpha}^{i, j k}+\Omega^{i k} \xi_{\alpha \beta \gamma}{ }^{j}+\Omega^{i j} \varepsilon_{\alpha \beta \gamma \delta} \tilde{\xi}^{\delta k}+(\underline{\alpha} \leftrightarrow \underline{\beta}) .
\end{aligned}
$$

Finally, at dimension 5 one finds

$$
4 \partial_{[a} H_{b c d]}=\frac{1}{1920}\left(\tilde{\gamma}_{[a}\right)^{\gamma \delta}\left(\tilde{\gamma}_{b c d]}\right)^{\alpha \beta} D_{\gamma k} D_{\delta l} H_{\alpha \beta}{ }^{k l}-\frac{1}{480}\left(\tilde{\gamma}_{[a}\right)^{\gamma \delta}\left(\tilde{\gamma}_{b c d]}\right)^{\alpha \beta} D_{\gamma k} D_{\alpha l} H_{\delta \beta}{ }^{k l} .
$$

All constraints on $H_{\alpha}^{i, j k}$ are encoded in the closure of the four-form $H_{4}$.

From the above results one can determine the components of the four-form:

$$
\begin{aligned}
H_{a b \alpha \beta}^{i j} & =-\frac{1}{2}\left(\gamma_{a b}\right)_{\alpha}{ }^{\gamma} H_{\beta \gamma}{ }^{j i}-\frac{1}{2}\left(\gamma_{a b}\right)_{\beta}{ }^{\gamma} H_{\alpha \gamma}{ }^{i j}, \\
H_{a b c \gamma} k & =\frac{\mathrm{i}}{240}\left(\tilde{\gamma}_{a b c}\right)^{\alpha \beta}\left(D_{\gamma j} H_{\alpha \beta}{ }^{k j}-4 D_{\alpha j} H_{\gamma \beta}{ }^{k j}\right), \\
H_{a b c d} & =\frac{1}{1920}\left(\tilde{\gamma}_{[a}\right)^{\gamma \delta}\left(\tilde{\gamma}_{b c d]}\right)^{\alpha \beta} D_{\gamma k} D_{\delta l} H_{\alpha \beta}{ }^{k l}-\frac{1}{480}\left(\tilde{\gamma}_{[a}\right)^{\gamma \delta}\left(\tilde{\gamma}_{b c d]}\right)^{\alpha \beta} D_{\gamma k} D_{\alpha l} H_{\delta \beta}{ }^{k l} .
\end{aligned}
$$

It is important to emphasise that all the differential constraints on $H_{\alpha \beta}{ }^{i j}$ can be projected out of the closure condition (D.7). However, we do not give them explicitly here for simplicity.

Open Access. This article is distributed under the terms of the Creative Commons Attribution License (CC-BY 4.0), which permits any use, distribution and reproduction in any medium, provided the original author(s) and source are credited.

\section{References}

[1] S. Ferrara and B. Zumino, Transformation Properties of the Supercurrent, Nucl. Phys. B 87 (1975) 207 [INSPIRE].

[2] Y. Korovin, S.M. Kuzenko and S. Theisen, The conformal supercurrents in diverse dimensions and conserved superconformal currents, JHEP 05 (2016) 134 [arXiv: 1604.00488] [INSPIRE].

[3] P.S. Howe and U. Lindström, The Supercurrent in Five-dimensions, Phys. Lett. B 103 (1981) 422 [INSPIRE].

[4] P.S. Howe, G. Sierra and P.K. Townsend, Supersymmetry in Six-Dimensions, Nucl. Phys. B 221 (1983) 331 [INSPIRE].

[5] S.J. Gates Jr., Super p-form gauge superfields, Nucl. Phys. B 184 (1981) 381 [INSPIRE].

[6] S.J. Gates, M.T. Grisaru, M. Roček and W. Siegel, Superspace Or One Thousand and One Lessons in Supersymmetry, Front. Phys. 58 (1983) 1 [hep-th/0108200] [InSPIRE].

[7] T.E. Clark, O. Piguet and K. Sibold, Supercurrents, Renormalization and Anomalies, Nucl. Phys. B 143 (1978) 445 [INSPIRE]. 
[8] M.F. Sohnius and P.C. West, An Alternative Minimal Off-Shell Version of $N=1$ Supergravity, Phys. Lett. B 105 (1981) 353 [InSPIRE].

[9] S.J. Gates Jr., M.T. Grisaru and W. Siegel, Auxiliary field anomalies, Nucl. Phys. B 203 (1982) 189 [INSPIRE].

[10] V. Ogievetsky and E. Sokatchev, On Vector Superfield Generated by Supercurrent, Nucl. Phys. B 124 (1977) 309 [INSPIRE].

[11] S. Ferrara and B. Zumino, Structure of Conformal Supergravity, Nucl. Phys. B 134 (1978) 301 [INSPIRE].

[12] W. Siegel, A derivation of the supercurrent superfield, Harvard preprint HUTP-77/A089 (1977).

[13] J. Wess and B. Zumino, Superfield Lagrangian for Supergravity, Phys. Lett. B 74 (1978) 51 [INSPIRE].

[14] K.S. Stelle and P.C. West, Minimal Auxiliary Fields for Supergravity, Phys. Lett. B 74 (1978) 330 [INSPIRE].

[15] S. Ferrara and P. van Nieuwenhuizen, The Auxiliary Fields of Supergravity, Phys. Lett. B 74 (1978) 333 [INSPIRE].

[16] S. Deser, Scale invariance and gravitational coupling, Annals Phys. 59 (1970) 248 [INSPIRE].

[17] B. Zumino, Effective Lagrangians and broken symmetries, in Lectures on Elementary Particles and Quantum Field Theory. Vol. 2, S. Deser, M. Grisaru and H. Pendleton eds., the M.I.T. Press, Cambridge U.S.A. (1970), pp. 437-500.

[18] P.A.M. Dirac, Long range forces and broken symmetries, Proc. Roy. Soc. Lond. A 333 (1973) 403 [INSPIRE].

[19] W. Siegel, A polynomial action for a massive, self-interacting chiral superfield coupled to supergravity, HUTP-77/A077 (1977).

[20] M. Kaku and P.K. Townsend, Poincaré supergravity as broken superconformal gravity, Phys. Lett. B 76 (1978) 54 [INSPIRE].

[21] R. Manvelyan and W. Rühl, On the supermultiplet of anomalous currents in D=6, Phys. Lett. B 567 (2003) 53 [hep-th/0305138] [INSPIRE].

[22] K.S. Stelle, Extended supercurrents and the ultraviolet finiteness of $N=4$ supersymmetric Yang-Mills theory, in Quantum Structure of Space and Time, M.J. Duff and C.J. Isham eds., Cambridge University Press, Cambridge (1982), pp. 337-361.

[23] M.F. Sohnius, The Multiplet of Currents for $N=2$ Extended Supersymmetry, Phys. Lett. B 81 (1979) 8 [INSPIRE].

[24] P.S. Howe, K.S. Stelle and P.K. Townsend, Supercurrents, Nucl. Phys. B 192 (1981) 332 [INSPIRE].

[25] S.M. Kuzenko and S. Theisen, Correlation functions of conserved currents in $N=2$ superconformal theory, Class. Quant. Grav. 17 (2000) 665 [hep-th/9907107] [INSPIRE].

[26] D. Butter and S.M. Kuzenko, N=2 supergravity and supercurrents, JHEP 12 (2010) 080 [arXiv: 1011.0339] [INSPIRE].

[27] A. Galperin, E. Ivanov, S. Kalitsyn, V. Ogievetsky and E. Sokatchev, Unconstrained $N=2$ Matter, Yang-Mills and Supergravity Theories in Harmonic Superspace, Class. Quant. Grav. 1 (1984) 469 [Erratum ibid. 2 (1985) 127] [INSPIRE]. 
[28] A.S. Galperin, E.A. Ivanov, V.I. Ogievetsky and E. Sokatchev, $N=2$ Supergravity in Superspace: Different Versions and Matter Couplings, Class. Quant. Grav. 4 (1987) 1255 [INSPIRE].

[29] A.S. Galperin, E.A. Ivanov, V.I. Ogievetsky and E.S. Sokatchev, Harmonic Superspace, Cambridge University Press, Cambridge (2001).

[30] S.V. Ketov and B.B. Lokhvitsky, Some Generalizations of $N=2$ Yang-Mills Matter Couplings, Class. Quant. Grav. 4 (1987) L137 [InSPIRE].

[31] S.V. Ketov, B.B. Lokhvitsky and I.V. Tyutin, HyperKähler $\sigma$ Models in Extended Superspace, Theor. Math. Phys. 71 (1987) 496 [Teor. Mat. Fiz. 71 (1987) 226] [InSPIRE].

[32] A.S. Galperin, E.A. Ivanov and V.I. Ogievetsky, Duality Transformations and Most General Matter Selfcoupling in N=2 Supersymmetry, Nucl. Phys. B 282 (1987) 74 [INSPIRE].

[33] U. Lindström and M. Roček, New HyperKähler Metrics and New Supermultiplets, Commun. Math. Phys. 115 (1988) 21 [inSPIRE].

[34] M.F. Sohnius, K.S. Stelle and P.C. West, Representations of extended supersymmetry, in Superspace and Supergravity, S.W. Hawking and M. Roček eds., Cambridge University Press, Cambridge (1981), p. 283-329.

[35] F. Gonzalez-Rey, M. Roček, S. Wiles, U. Lindström and R. von Unge, Feynman rules in $N=2$ projective superspace: 1. Massless hypermultiplets, Nucl. Phys. B 516 (1998) 426 [hep-th/9710250] [INSPIRE].

[36] W.D. Linch, III and G. Tartaglino-Mazzucchelli, Six-dimensional Supergravity and Projective Superfields, JHEP 08 (2012) 075 [arXiv: 1204.4195] [INSPIRE].

[37] S.M. Kuzenko, On compactified harmonic/projective superspace, 5-D superconformal theories and all that, Nucl. Phys. B 745 (2006) 176 [hep-th/0601177] [INSPIRE].

[38] S.M. Kuzenko, On superconformal projective hypermultiplets, JHEP 12 (2007) 010 [arXiv:0710.1479] [INSPIRE].

[39] S.M. Kuzenko and G. Tartaglino-Mazzucchelli, Super-Weyl invariance in $5 D$ supergravity, JHEP 04 (2008) 032 [arXiv:0802.3953] [INSPIRE].

[40] S.M. Kuzenko, U. Lindström, M. Roček and G. Tartaglino-Mazzucchelli, On conformal supergravity and projective superspace, JHEP 08 (2009) 023 [arXiv: 0905.0063] [INSPIRE].

[41] D. Butter, S.M. Kuzenko, J. Novak and S. Theisen, Invariants for minimal conformal supergravity in six dimensions, JHEP 12 (2016) 072 [arXiv: 1606. 02921] [INSPIRE].

[42] S.M. Kuzenko, J. Novak and I.B. Samsonov, The anomalous current multiplet in $6 D$ minimal supersymmetry, JHEP 02 (2016) 132 [arXiv: 1511.06582] [INSPIRE].

[43] S.M. Kuzenko, J. Novak and I.B. Samsonov, Chiral anomalies in six dimensions from harmonic superspace, JHEP 11 (2017) 145 [arXiv:1708.08238] [INSPIRE].

[44] E.A. Ivanov, A.V. Smilga and B.M. Zupnik, Renormalizable supersymmetric gauge theory in six dimensions, Nucl. Phys. B 726 (2005) 131 [hep-th/0505082] [INSPIRE].

[45] J. Grundberg and U. Lindström, Actions for Linear Multiplets in Six-dimensions, Class. Quant. Grav. 2 (1985) L33 [INSPIRE].

[46] E. Bergshoeff, E. Sezgin and A. Van Proeyen, Superconformal Tensor Calculus and Matter Couplings in Six-dimensions, Nucl. Phys. B 264 (1986) 653 [Erratum ibid. B 598 (2001) 667] [INSPIRE]. 
[47] P.S. Howe, K.S. Stelle and P.K. Townsend, The Relaxed Hypermultiplet: An Unconstrained $N=2$ Superfield Theory, Nucl. Phys. B 214 (1983) 519 [INSPIRE].

[48] C. Arias, W.D. Linch, III and A.K. Ridgway, Superforms in six-dimensional superspace, JHEP 05 (2016) 016 [arXiv: 1402.4823] [INSPIRE].

[49] S.M. Kuzenko, J. Novak and S. Theisen, New superconformal multiplets and higher derivative invariants in six dimensions, Nucl. Phys. B 925 (2017) 348 [arXiv: 1707. 04445] [INSPIRE].

[50] E. Sokatchev, Off-shell Six-dimensional Supergravity in Harmonic Superspace, Class. Quant. Grav. 5 (1988) 1459 [INSPIRE].

[51] P.S. Howe and A. Umerski, Anomaly multiplets in six-dimensions and ten-dimensions, Phys. Lett. B 198 (1987) 57 [INSPIRE].

[52] F. Coomans and A. Van Proeyen, Off-shell $N=(1,0), D=6$ supergravity from superconformal methods, JHEP 02 (2011) 049 [Erratum ibid. 01 (2012) 119] [arXiv:1101.2403] [InSPIRE].

[53] D. Butter, J. Novak and G. Tartaglino-Mazzucchelli, The component structure of conformal supergravity invariants in six dimensions, JHEP 05 (2017) 133 [arXiv:1701.08163] [INSPIRE].

[54] E. Bergshoeff, F. Coomans, E. Sezgin and A. Van Proeyen, Higher Derivative Extension of 6D Chiral Gauged Supergravity, JHEP 07 (2012) 011 [arXiv:1203.2975] [INSPIRE].

[55] L. Bonora, P. Pasti and M. Tonin, Cohomologies and Anomalies in Supersymmetric Theories, Nucl. Phys. B 252 (1985) 458 [InSPIRE].

[56] I.L. Buchbinder and S.M. Kuzenko, Matter Superfields in External Supergravity: Green Functions, Effective Action and Superconformal Anomalies, Nucl. Phys. B 274 (1986) 653 [INSPIRE].

[57] S.M. Kuzenko, Super-Weyl anomalies in $N=2$ supergravity and (non)local effective actions, JHEP 10 (2013) 151 [arXiv: 1307.7586] [INSPIRE].

[58] A. Karlhede, U. Lindström and M. Roček, Selfinteracting Tensor Multiplets in $N=2$ Superspace, Phys. Lett. B 147 (1984) 297 [INSPIRE].

[59] U. Lindström and M. Roček, $N=2$ Super Yang-Mills Theory in Projective Superspace, Commun. Math. Phys. 128 (1990) 191 [INSPIRE].

[60] S.M. Kuzenko and W.D. Linch, III, On five-dimensional superspaces, JHEP 02 (2006) 038 [hep-th/0507176] [INSPIRE].

[61] S.J. Gates Jr., S. Penati and G. Tartaglino-Mazzucchelli, 6D supersymmetry, projective superspace and $4 D, N=1$ superfields, JHEP 05 (2006) 051 [hep-th/0508187] [INSPIRE].

[62] S.J. Gates Jr., S. Penati and G. Tartaglino-Mazzucchelli, 6D Supersymmetric Nonlinear $\sigma$-models in 4D, $N=1$ Superspace, JHEP 09 (2006) 006 [hep-th/0604042] [INSPIRE].

[63] D. Butter, S.M. Kuzenko, J. Novak and G. Tartaglino-Mazzucchelli, Conformal supergravity in five dimensions: New approach and applications, JHEP 02 (2015) 111 [arXiv:1410.8682] [INSPIRE].

[64] D. Butter, New approach to curved projective superspace, Phys. Rev. D 92 (2015) 085004 [arXiv: 1406.6235] [INSPIRE].

[65] D. Butter, Projective multiplets and hyperkähler cones in conformal supergravity, JHEP 06 (2015) 161 [arXiv:1410.3604] [INSPIRE]. 
[66] E. Bergshoeff, E. Sezgin and E. Sokatchev, Couplings of selfdual tensor multiplet in six-dimensions, Class. Quant. Grav. 13 (1996) 2875 [hep-th/9605087] [INSPIRE].

[67] C. Grojean and J. Mourad, Superconformal 6-D (2,0) theories in superspace, Class. Quant. Grav. 15 (1998) 3397 [hep-th/9807055] [INSPIRE].

[68] P. Arvidsson, E. Flink and M. Henningson, Supersymmetric coupling of a selfdual string to a (2,0) tensor multiplet background, JHEP 11 (2003) 015 [hep-th/0309244] [INSPIRE]. 